\title{
The Dynamics of Energy Dissipation and Xanthophyll Conversion in Arabidopsis Indicate an Indirect Photoprotective Role of Zeaxanthin in Slowly Inducible and Relaxing Components of Non-photochemical Quenching of Excitation Energy
}

\author{
Eugen Kress and Peter Jahns* \\ Plant Biochemistry, Heinrich-Heine-University Düsseldorf, Düsseldorf, Germany
}

OPEN ACCESS

Edited by:

Benoit Schoefs,

University of Maine, France

Reviewed by:

Radek Kana,

Institute of Microbiology, Czech Academy of Sciences, Czechia

David Kramer,

Michigan State University,

United States

*Correspondence:

Peter Jahns

pjahns@hhu.de

Specialty section:

This article was submitted to

Plant Physiology,

a section of the journal

Frontiers in Plant Science

Received: 20 July 2017 Accepted: 24 November 2017 Published: 08 December 2017

Citation:

Kress E and Jahns P (2017) The Dynamics of Energy Dissipation and Xanthophyll Conversion in Arabidopsis Indicate an Indirect Photoprotective Role of Zeaxanthin in Slowly Inducible and Relaxing Components of Non-photochemical Quenching of Excitation Energy.

Front. Plant Sci. 8:2094. doi: 10.3389/fp/s.2017.02094
The dynamics of non-photochemical quenching (NPQ) of chlorophyll fluorescence and the dynamics of xanthophyll conversion under different actinic light conditions were studied in intact leaves of Arabidopsis thaliana. NPQ induction was investigated during up to 180 min illumination at 450, 900, and 1,800 $\mu$ mol photons $\mathrm{m}^{-2} \mathrm{~s}^{-1}(\mu \mathrm{E})$ and NPQ relaxation after $5,30,90$, or 180 min of pre-illumination at the same light intensities. The comparison of wild-type plants with mutants affected either in xanthophyll conversion (npq1 and npq2) or PsbS expression (npq4 and L17) or lumen acidification (pgr1) indicated that NPQ states with similar, but not identical characteristics are induced at longer time range (15-60 min) in wild-type and mutant plants. In genotypes with an active xanthophyll conversion, the dynamics of two slowly (10-60 min) inducible and relaxing $\mathrm{NPQ}$ components were found to be kinetically correlated with zeaxanthin formation and epoxidation, respectively. However, the extent of NPQ was independent of the amount of zeaxanthin, since higher NPQ values were inducible with increasing actinic light intensities without pronounced changes in the zeaxanthin amount. These data support an indirect role of zeaxanthin in $\mathrm{pH}$-independent NPQ states rather than a specific direct function of zeaxanthin as quencher in long-lasting NPQ processes. Such an indirect function might be related to an allosteric regulation of NPQ processes by zeaxanthin (e.g., through interaction of zeaxanthin at the surface of proteins) or a general photoprotective function of zeaxanthin in the lipid phase of the membrane (e.g., by modulation of the membrane fluidity or by acting as antioxidant). The found concomitant down-regulation of zeaxanthin epoxidation and recovery of photosystem II activity ensures that zeaxanthin is retained in the thylakoid membrane as long as photosystem II activity is inhibited or down-regulated. This regulation supports the view that zeaxanthin can be considered as a kind of light stress memory in chloroplasts, allowing a rapid reactivation of photoprotective NPQ processes in case of recurrent light stress periods.

Keywords: energy dissipation, non-photochemical quenching, photoinhibition, photosynthesis, xanthophyll cycle, zeaxanthin 


\section{INTRODUCTION}

Sunlight is not only the ultimate energy source for photosynthesis, but also the major source for the formation of reactive oxygen species (ROS) in chloroplasts. The latter is related to the fact that plants frequently absorb more light than they can use in photosynthesis. To cope with the damaging potential of excess light energy, plants have developed a number of strategies (i) to reduce the absorption of light (e.g., chloroplast movement, reduction of the antenna size), (ii) to reduce the amount of ROS production (e.g., alternative electron pathways, heat dissipation), or (iii) to detoxify produced ROS by antioxidants and antioxidative enzymes ( $\mathrm{Li}$ et al., 2009). In the field, light intensities may vary in orders of magnitude at short time scale (seconds to minutes), which determines the demand for a flexible photoprotective response in the short-term. The dissipation of excess light as heat, also known as non-photochemical quenching (NPQ) of excitation energy, matches all requirements of such a flexible response to excess light. The overall NPQ is complex and comprises at least four different components, which have been termed (1) $\mathrm{qE}$, the $\mathrm{pH}$-regulated energy dissipation in the antenna of photosystem II (PSII) (Krause et al., 1982), (2) qT, state transitions (Allen et al., 1981), (3) qZ, zeaxanthin-dependent quenching (Nilkens et al., 2010), and (4) qI, photoinhibition (Krause, 1988). The relative contribution of the different components, which act on different time scales, to the total NPQ is variable and primarily depends, at least for plants grown under controlled lab conditions, on the illumination intensity and time.

\section{Characteristics of NPQ components}

The $\mathrm{qE}$ component is the most rapidly $(1-3 \mathrm{~min})$ inducible and relaxing component, which is essentially controlled by the thylakoid lumen $\mathrm{pH}$. Drop of the lumen $\mathrm{pH}$ below about $\mathrm{pH}$ 6.0 activates the PsbS protein ( $\mathrm{Li}$ et al., 2000, 2002) which is supposed to induce conformational changes in the lightharvesting antenna of PSII (LHCII) through direct interaction with LHCII proteins (Correa-Galvis et al., 2016; Sacharz et al., 2017). $\mathrm{qE}$ is further modulated by the xanthophyll zeaxanthin $(\mathrm{Zx})$ which is synthesized from violaxanthin $(\mathrm{Vx})$ in the deepoxidation reactions of the xanthophyll cycle (Jahns et al., 2009). Like qE activation through PsbS, also the formation of $\mathrm{Zx}$ is controlled by the lumen $\mathrm{pH}$, which occurs at $\mathrm{pH}$ values $<6$ by activation of the lumen-localized Vx de-epoxidase (Hager, 1969). This $\mathrm{pH}$ regulation of $\mathrm{qE}$ allows a flexible adjustment of energy dissipation in response to fluctuating light intensities, and ensures that the dissipation of absorbed light energy is not active under light-limiting conditions, but only upon lightsaturation of photosynthetic electron transport, as indicated by the lumen $\mathrm{pH}$. The importance of the rapid down-regulation of $\mathrm{qE}$ after a transition from high light to low light, as given under fluctuating light, has been proven recently for plants with altered qE relaxation properties (Armbruster et al., 2014; Kromdijk et al., 2016).

The qT component of NPQ operates in the time scale of 10-20 min and serves the optimal balancing of the energy distribution between PSII and PSI (Allen, 2003). Activation of
qT is triggered by the reduction of the plastoquinone pool through activation of the kinase STT7 (Chlamydomonas) or STN7 (Arabidopsis) (Bellafiore et al., 2005; Bonardi et al., 2005), which phosphorylates LHCII proteins. Phosphorylated LHCII is thought to detach from PSII and to associate with PSI, which decreases the functional antenna size of PSII and increases that of PSI. This process is reversible upon dephosphorylation of LHCII by the phosphatase TAP38/PPH1 (Pribil et al., 2010; Shapiguzov et al., 2010), which is particularly activated at high light. In land plants, the contribution of qT to the total NPQ is generally low and no significant contribution at all has been observed under saturating light conditions in Arabidopsis (Nilkens et al., 2010).

The $\mathrm{qZ}$ component of NPQ operates on a time scale from 10 to $30 \mathrm{~min}$ and has been assigned to $\mathrm{Zx}$ on basis of its kinetic correlation with the reconversion of $\mathrm{Zx}$ to $\mathrm{Vx}$ (Nilkens et al., 2010). In contrast to $\mathrm{qE}, \mathrm{qZ}$ remains active in absence of a transthylakoid proton gradient (Dall'Osto et al., 2005) and thus in darkness. This NPQ component is likely identical with the component, which was earlier ascribed to a fast relaxing component of photoinhibition (Leitsch et al., 1994; Jahns and Miehe, 1996; Thiele et al., 1998).

The qI component of NPQ comprises all processes that are directly related to the photoinhibition of PSII. Photoinhibition of PSII (and thus activation of qI) typically occurs upon high light illumination at a time scale of hours, but maybe induced also at shorter time when photosynthesis is limited by unfavorable environmental conditions such as drought or low temperature. Photoinhibition of PSII is based on the damage of the D1 protein of the PSII reaction center (Aro et al., 1993). Recovery from photoinhibition requires the degradation of damaged D1 and its replacement by newly synthesized D1 protein. The PSII inhibition-repair cycle involves reversible phosphorylation of PSII reaction center proteins. In its phosphorylated state, damaged PSII monomerizes and moves from the grana region of the thylakoid membrane to stroma-exposed regions, where the repair of damaged D1 is supposed to take place (Tikkanen and Aro, 2012). Under most conditions, the degradation of damaged D1 protein is fully compensated by repair processes (Kyle et al., 1984), so that a net degradation of D1 occurs only under extreme light stress conditions or when the synthesis of D1 is blocked by inhibitors of protein synthesis in chloroplasts (Samuelsson et al., 1985). Hence, long-term exposure of plants to high light intensities involves the permanent repair of PSII and thus requires a highly flexible reorganization of the thylakoid membrane.

\section{Role of Zx in Energy Dissipation Processes}

The light-dependent and reversible conversion of $\mathrm{Vx}$ to $\mathrm{Zx}$ in plants has been discovered more than 50 years ago (Sapozhnikov et al., 1957; Yamamoto et al., 1962). This so-called violaxanthin cycle is known to be active in all land plants, brown algae and most of the green algae, while the diadinoxanthin cycle, which involves the reversible conversion of diadinoxanthin ( $\mathrm{Ddx}$ ) to diatoxanthin (Dtx) is active in a wide range of different algae including diatoms and haptophytes (for reviews see (Jahns et al., 2009; Goss and Jakob, 2010). The wide distribution of light-dependent xanthophyll conversion concomitant with 
the activation of NPQ supports an important photoprotective function of xanthophylls. This view is further supported by the fact, that also in photosynthetic organisms, which do not possess an active xanthophyll cycle, such as cyanobacteria, xanthophylls are supposed to serve central functions in energy dissipation (Wilson et al., 2006; Leverenz et al., 2015). It is a matter of a long lasting debate, however, whether the photoprotective function of xanthophylls is based on a direct function as quencher of excitation energy or an indirect function as modulator or regulator of energy dissipation, or as antioxidant in the lipid phase. These different modes of action depend on the respective localization of $\mathrm{Zx}$. A direct function of $\mathrm{Zx}$ as quencher requires the binding of $\mathrm{Zx}$ at specific xanthophyll binding sites in antenna proteins. An indirect function of Zx likely involves an allosteric regulation of NPQ (Horton et al., 2000), which might be induced by either the release of $\mathrm{Vx}$ from xanthophyll binding sites (Kana et al., 2016) or the exchange of $\mathrm{Vx}$ by $\mathrm{Zx}$ at a xanthophyll binding site (Ruban and Horton, 1999; Kana et al., 2016), or through interaction of $\mathrm{Zx}$ with antenna proteins at the lipid-protein interface (Xu et al., 2015). Finally, a more general photoprotective function of $\mathrm{Zx}$ has been proposed for $\mathrm{Zx}$ in the lipid phase of the thylakoid membrane, either as modulator of membrane properties (Havaux, 1998) or as antioxidant (Havaux et al., 2004).

In the case of $\mathrm{Zx}$, a function in energy dissipation has been proposed some 30 years ago (Demmig et al., 1987; Demmig-Adams, 1990; Demmig-Adams and Adams, 1996). This hypothesis was finally proven by the identification and characterization of Arabidopsis xanthophyll cycle mutants $n p q 1$ and $n p q 2$, which are defective in the xanthophyll cycle enzymes Vx de-epoxidase (VDE) and Zx epoxidase (ZEP), respectively (Niyogi et al., 1998). The Zx-deficient npq1 mutant exhibits a markedly reduced $\mathrm{qE}$ capacity, although a significant part of $\mathrm{qE}$ is still inducible in this mutant (Nilkens et al., 2010). In contrast, the $\mathrm{Zx}$ accumulating $n p q 2$ mutant is particularly characterized by a more rapidly inducible and more slowly relaxing $\mathrm{qE}$ (Niyogi et al., 1998; Dall'Osto et al., 2005; Nilkens et al., 2010). It is still unclear whether Zx acts as a direct quencher of excitation energy or whether Zx functions in an indirect manner as modulator of energy dissipation (in case of $\mathrm{qE}$ ) or simply as photoprotective component in the lipid phase of the membrane (in case of longlasting NPQ states, such as $\mathrm{qZ}$ and $\mathrm{qI}$ ). Evidence for a direct energy quenching by $\mathrm{Zx}$ has been derived so far only from timeresolved transient absorption measurements performed with isolated thylakoid membranes (Holt et al., 2005) or isolated antenna complexes (Ahn et al., 2008; Avenson et al., 2008). Based on these analyses the formation of a Chl-Zx charge transfer complex has been proposed to occur in minor antenna complexes (Lhcb4-6), but not in the major trimeric antenna complexes (LHCII) of PSII. However, recent analysis of minor and major antenna complexes isolated from dark-adapted (no $\mathrm{Zx}$ ) or preilluminated (with $\mathrm{Zx}$ ) plants, could not provide evidence for binding of significant amounts of $\mathrm{Zx}$ to single complexes, nor for any differences in the quenching capacity of the complexes in absence and presence of Zx (Xu et al., 2015).

While possible quenching sites and mechanisms have been studied in detail to clarify the role of $\mathrm{Zx}$ in $\mathrm{qE}$, nearly no information is available for the role of $\mathrm{Zx}$ in more slowly relaxing NPQ components ( $\mathrm{qZ}$ and $\mathrm{qI}$ ), which remain active after relaxation of the transthylakoid $\mathrm{pH}$ gradient. Evidence for a role of $\mathrm{Zx}$ in $\mathrm{qZ}$ and $\mathrm{qI}$ has mainly been derived from the correlation of the dynamics of the $\mathrm{Zx}$ content and the dynamics of NPQ states. In fact, the $\mathrm{qZ}$ component of NPQ has been identified by the close correlation of the medium phase of NPQ relaxation and Zx epoxidation (Nilkens et al., 2010). Moreover, sustained inactivation of PSII induced by long-lasting high light stress has been correlated with sustained retainment of $\mathrm{Zx}$ in numerous studies (Ebbert et al., 2001, 2005; Adams et al., 2002; Demmig-Adams et al., 2006, 2012). Though such approaches are limited by the missing information about the underlying quenching mechanism, they can provide important information about the possible role of $\mathrm{Zx}$ in quenching. Here, we extended the investigation of the correlation of $\mathrm{Zx}$ and NPQ dynamics by comparing both processes at different light intensities, which induce to different extent the NPQ components $\mathrm{qE}, \mathrm{qZ}$ and $\mathrm{qI}$ in Arabidopsis leaves. The use of different mutants affected in PsbS levels ( $n p q 4$ and L17), the xanthophyll cycle (npq1 and npq2) and lumen acidification ( $p g r 1)$ allowed us to correlate particularly the dynamics of the two slowly inducible and relaxing components $\mathrm{qZ}$ and $\mathrm{qI}$ with the dynamics of xanthophyll conversion. We show that NPQ components with similar characteristics of $\mathrm{qZ}$ and $\mathrm{qI}$ are formed in all genotypes although the $\mathrm{Zx}$ content varied largely among the genotypes. However, in genotypes with an active xanthophyll cycle, the reconversion of $\mathrm{Zx}$ to $\mathrm{Vx}$ was found to be closely correlated with the relaxation of slowly relaxing NPQ states. Based on these results, we hypothesize that Zx has no direct quenching function in $\mathrm{qZ}$ and $\mathrm{qI}$, but rather functions as indirect regulator or photoprotective molecule in NPQ states that remain active in absence of a low lumen $\mathrm{pH}$ after moderate or severe high light stress.

\section{MATERIALS AND METHODS Plant Material and Growth Conditions}

Arabidopsis thaliana wild-type (ecotype Col-0) plants and the mutant plants npq4 (Li et al., 2000), L17 (Li et al., 2002), npq1 and npq2 (Niyogi et al., 1998), and pgr1 (Munekage et al., 2001) were grown on soil at a light intensity of $150 \mu \mathrm{mol}$ photons $\mathrm{m}^{-2} \mathrm{~s}^{-1}$ and a constant temperature of $20^{\circ} \mathrm{C}$ under long-day conditions ( $14 \mathrm{~h}$ light $/ 10 \mathrm{~h}$ dark). Leaves from 5 to 6 weeks-old plants were used for all experiments.

\section{Chlorophyll Fluorescence Measurements}

$\mathrm{Chl}$ a fluorescence was measured with detached leaves at $20^{\circ} \mathrm{C}$ with a pulse-amplitude-modulated fluorometer (PAM 101, Walz, Effeltrich, Germany). Leaves were placed on wet filter paper in a self-built cuvette, which allowed the control of the leaf temperature and the supply with moistened air throughout the experiment. For all experiments, leaves were dark-adapted for at least $2 \mathrm{~h}$ before start of the experiment. For NPQ induction, leaves were illuminated with white light for up to $180 \mathrm{~min}$ at light intensities of 450,900 , or $1,800 \mu \mathrm{mol}$ photons $\mathrm{m}^{-2}$ $\mathrm{s}^{-1}$. Relaxation of NPQ was determined during a subsequent dark phase for up to $180 \mathrm{~min}$. For the determination of NPQ induction, saturating white light pulses 4,000 $\mu \mathrm{mol}$ photons 
$\mathrm{m}^{-2} \mathrm{~s}^{-1}$, duration $800 \mathrm{~ms}$ were applied every $20 \mathrm{~s}$ during the first $100 \mathrm{~s}$ of illumination and every $100 \mathrm{~s}$ for the remaining illumination time. For the determination of NPQ relaxation, saturating light pulses were spaced $20 \mathrm{~s}$ for the first $100 \mathrm{~s}$, followed by 8 flashes spaced $100 \mathrm{~s}$ and flashes spaced $500 \mathrm{~s}$ for the remaining time. Control measurements in absence of actinic light indicated that the saturating light pulses alone neither induced reasonable NPQ $(>0.02)$ during the induction of NPQ, nor affected the NPQ relaxation kinetics. NPQ was determined as (Fm/Fm'-1) (Krause and Jahns, 2003).

\section{Correlation Analyses}

The correlation of NPQ and Zx was evaluated by linear regression analysis of plots of NPQ values vs. the Zx content. Regression analysis was performed with Grafit software (Grafit 5, Erithacus Software Limited, UK) and the resulting Pearson's correlation coefficients were determined.

\section{Pigment Analysis}

To determine the formation of $\mathrm{Zx}$, detached leaves from darkadapted plants were floated on water and illuminated at the same intensities of white light as used for NPQ induction for up to $180 \mathrm{~min}$. Reconversion of $\mathrm{Zx}$ to $\mathrm{Vx}$ was induced by transfer of pre-illuminated leaves to darkness. At the indicated times, leaves were frozen in liquid $\mathrm{N}_{2}$ and stored at $-80^{\circ} \mathrm{C}$. Pigments were extracted with acetone and quantified by reverse phase HPLC as described earlier (Färber et al., 1997).

\section{RESULTS}

We determined the dynamics of NPQ induction and relaxation in six genotypes and at three different actinic light (AL) intensities of 450,900 , and $1,800 \mu \mathrm{mol}$ photons $\mathrm{m}^{-2} \mathrm{~s}^{-1}$ in comparison with the dynamics of $\mathrm{Zx}$ synthesis and reconversion. The overall data are summarized as an overview in Figure S1 (NPQ dynamics) and Figure S2 (Zx dynamics). For all experiments, dark-adapted leaf discs were illuminated for up to $180 \mathrm{~min}$ to determine NPQ induction and Zx synthesis, whereas dark relaxation of NPQ and $\mathrm{Zx}$ reconversion was measured for up to $180 \mathrm{~min}$ after four different times of pre-illumination (5, 30, 90, and $180 \mathrm{~min}$ ). This experimental setup was chosen to determine in particular the characteristics of the slowly developing and relaxing NPQ components $\mathrm{qZ}$ and $\mathrm{qI}$ in comparison with $\mathrm{Zx}$ dynamics. The three AL intensities (for the sake of simplicity, the unit $\mu \mathrm{mol}$ photons $\mathrm{m}^{-2} \mathrm{~s}^{-1}$ will be abbreviated as $\mu \mathrm{E}$ throughout the text and all figures) represent illumination conditions, which are expected to either induce predominantly the $\mathrm{qE}$ component only $(450 \mu \mathrm{E})$ or both the $\mathrm{qE}$ and $\mathrm{qZ}$ components $(900 \mu \mathrm{E})$ or additionally pronounced photoinhibitory qI quenching $(1,800$ $\mu \mathrm{E})$. Wild-type (WT) plants were analyzed in comparison with mutants affected in the major regulators of NPQ, namely the lumen $\mathrm{pH}$ (pgr1), the xanthophyll cycle ( $n p q 1$ and $n p q 2)$, and the PsbS protein (npq4 and L17). In general, the data supported the well-known differences among the different genotypes. Pronounced rapid (within few min) $\mathrm{pH}$-dependent NPQ induction and relaxation was only detectable in WT, L17, and $n p q 2$ plants, while the absence or strong reduction of rapid
NPQ changes in the other genotypes is related to limited lumen acidification ( $p g r 1)$, the absence of PsbS ( $n p q 4)$, or the absence of $\mathrm{Zx}(n p q 1)$ (Figure S1). Zx formation was detectable with similar characteristics in all genotypes with an active xanthophyll cycle, except for pgrl plants, which showed a delayed and reduced Zx synthesis (Figure S1), reflecting the limited lumen acidification in this mutant. In the following, the characteristics of the NPQ dynamics are presented and explained in more detail, and further compared with the $\mathrm{Zx}$ dynamics. The $\mathrm{pH}$-regulated $\mathrm{qE}$ component is denoted as rapidly inducible/relaxing NPQ component, while the remaining NPQ processes are termed as slowly inducible/relaxing NPQ components, comprising $\mathrm{qZ}$ and qI. It should be noted, that the qT component of NPQ, which is related to state transitions, is not included in the slow NPQ components, because former analysis of the qT-deficient $\sin 7$ mutant has shown that $\mathrm{qT}$ does not contribute significantly to NPQ in Arabidopsis under our experimental conditions (Nilkens et al., 2010).

\section{NPQ Induction at Different AL Intensities}

The NPQ induction at the three different AL intensities is illustrated in Figure 1. It is obvious that the genotypes differ predominantly in the induction of rapid NPQ processes, which reflect the $\mathrm{pH}$-regulated $\mathrm{qE}$ component of NPQ. As expected, no rapid NPQ induction was detectable at all AL intensities in pgr1 (Figure 1B) and npq4 (Figure 1D) plants, underlining the known essential role of the lumen $\mathrm{pH}$ (Briantais et al., 1979) and PsbS (Li et al., 2000), respectively, for $\mathrm{qE}$ activation. The partial reduction of $\mathrm{qE}$ in the $n p q 1$ (Figure 1F) mutant supports the idea that $\mathrm{Zx}$ modulates the $\mathrm{qE}$ capacity, but is not an essential prerequisite for $\mathrm{qE}$ (Horton et al., 2000; Nilkens et al., 2010). Comparing the impact of the different $\mathrm{AL}$ intensities on $\mathrm{qE}$ induction in the three genotypes with high $\mathrm{qE}$ capacity (WT, L17, npq2) it is apparent that almost the maximum $\mathrm{qE}$ capacity is induced at the lowest intensity of $450 \mu \mathrm{E}$ (Figures 1A,C,E). This indicates that the lumen $\mathrm{pH}$ induced at the lowest AL intensity is already low enough to nearly saturate $\mathrm{qE}$ induction. Consequently, the increase of the AL intensity to 900 and $1,800 \mu \mathrm{E}$ predominantly leads to the additional induction of the slowly activated NPQ components qZ and qI (Figure 1).

In contrast to $\mathrm{qE}$, however, these two slowly inducible NPQ components were generated to a similar extent in all genotypes, except for the $L 17$ mutant (Figure 1C), which revealed a more pronounced increase of NPQ than all other genotypes. For a better evaluation of the increase of NPQ induced by increasing $\mathrm{AL}$ intensities, we plotted for each genotype the differences of the NPQ induction curves determined at the different AL intensities (900 minus $450 \mu \mathrm{E}$ or 1,800 minus $900 \mu \mathrm{E}$; Figure 2). The increase of the AL intensity from 450 to $900 \mu \mathrm{E}$ induced an increase of the maximum NPQ level by about 1.2 in L17 plants (Figure 2C), but only by about 0.5 in all other genotypes (Figure 2). In plants with high $\mathrm{qE}$ capacity (WT, $L 17$ and $n p q 2$ ), the increased NPQ capacity developed faster (half-rise time about $5 \mathrm{~min}$ ) than in the other genotypes (half-rise time about $15 \mathrm{~min}$ ). This phase has earlier been assigned to the activation of $\mathrm{qZ}$ quenching, but the presence of this component in $n p q 2$ and $n p q 1$ plants indicates that a similar NPQ component is inducible 


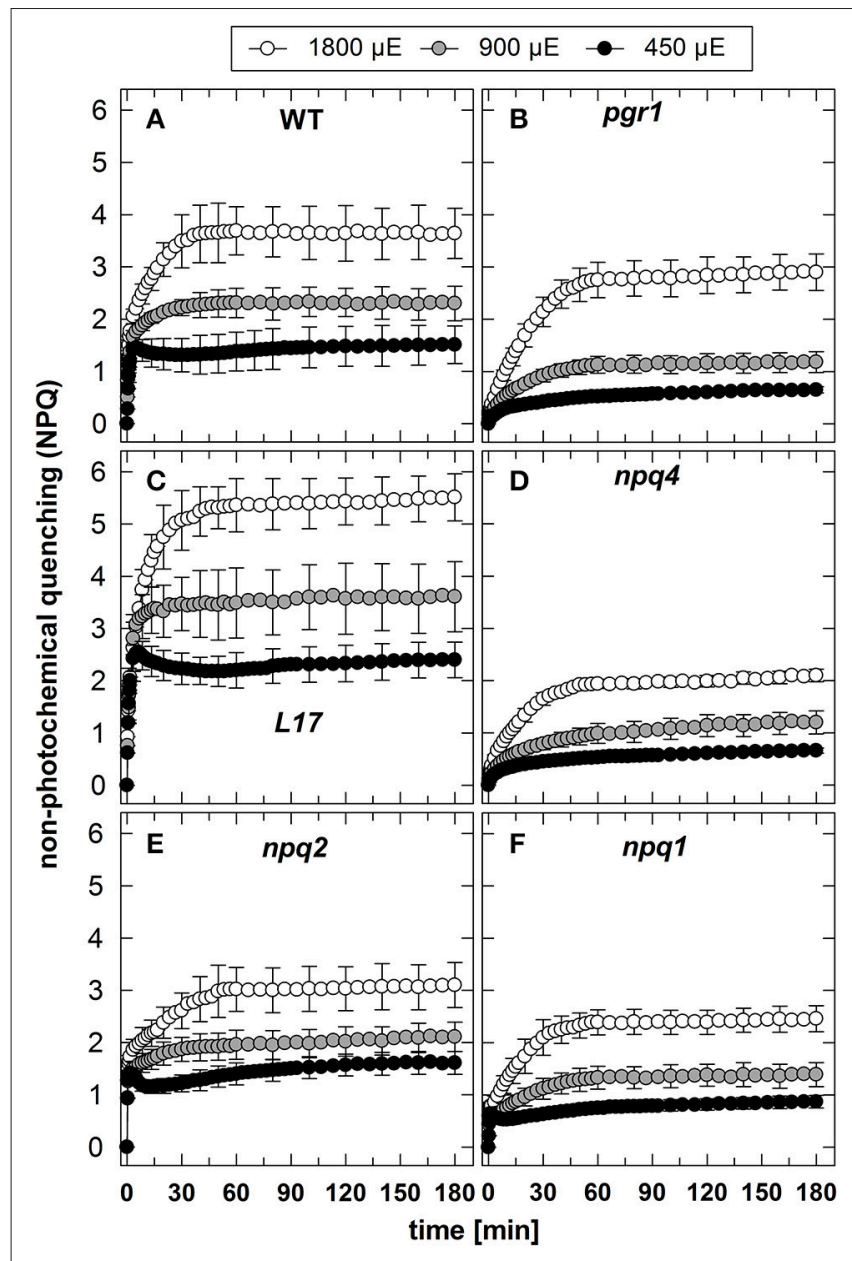

FIGURE 1 | NPQ induction. The induction of NPQ during 180 min of illumination at three different actinic light intensities $(450,900$, and 1,800 $\mu \mathrm{E}$ of white light) was determined for (A) WT, (B) pgr1, (C) L17, (D) npq4, (E), npq2, and (F) npq1 plants. During the whole measurements, detached leaves were placed on wet paper in a temperature-controlled cuvette $\left(20^{\circ} \mathrm{C}\right)$ under permanent supply with ambient air. Mean values \pm SE of 3-6 independent measurements are shown.

independent of $\mathrm{Zx}$. The increase of the AL intensity from 900 to $1,800 \mu \mathrm{E}$ induced a further increase of the maximum NPQ level in all genotypes. This increase can be predominantly assigned to the qI component of NPQ and occurred with similar kinetics in all genotypes but with different amplitudes in the range from about 1.0 (npq1, npq2, and npq4) to about 1.5-2.0 (WT, pgr1, and L17) (see Figure 2). Hence, the induction of the slowly developing NPQ components, qZ and qI, showed quite similar characteristics in all genotypes, and is thus independent of the lumen $\mathrm{pH}$, the PsbS protein and $\mathrm{Zx}$ synthesis.

\section{Zx Synthesis at Different AL Intensities}

The conversion of $\mathrm{Vx}$ to $\mathrm{Zx}$ during illumination (Figure 3) was determined under the same experimental conditions, aside from the fact that no saturating flashes were applied. Since no changes in the $\mathrm{Zx}$ content was detectable for the two xanthophyll cycle mutants $n p q 1(0 \% \mathrm{Zx})$ and $n p q 2(100 \% \mathrm{Zx})$, no data are shown

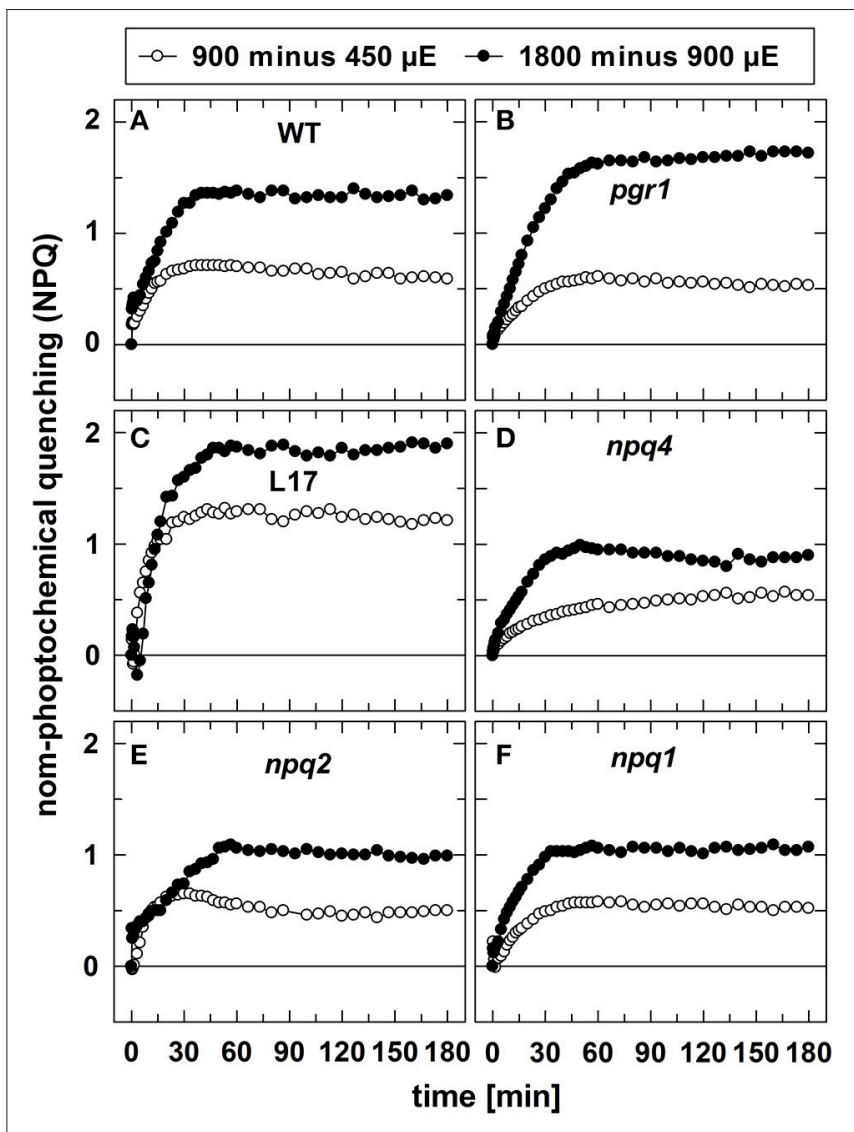

FIGURE 2 | Differences in NPQ induction upon increase of light intensities. To visualize the differences in NPQ induction upon increase of the actinic light intensity from either 450 to $900 \mu \mathrm{E}$ or from 900 to $1,800 \mu \mathrm{E}$, the differences of the respective curves shown in in Figure $\mathbf{1}$ were plotted for all genotypes. (A) WT, (B) pgr1, (C) L17, (D) npq4, (E), npq2, and (F) npq1 plants.

for these two genotypes. For WT (Figure 3A), L17 (Figure 3C), and $n p q 4$ (Figure 3D) plants, both the extent and the kinetics of $\mathrm{Zx}$ synthesis were very similar at all $\mathrm{AL}$ intensities, indicating that $\mathrm{Vx}$ de-epoxidation characteristics are independent of the PsbS protein. Moreover, the maximum $\mathrm{Zx}$ content was only slightly increased upon increase of the AL intensity from 450 to 900 and $1,800 \mu \mathrm{E}$. This suggests that the light-induced lumen acidification is close to saturation of the VDE activity already at the lowest $\mathrm{AL}$ intensity, in accordance with the observed characteristics of $\mathrm{qE}$ (Figure 2). Compared to these genotypes, however, the formation of $\mathrm{Zx}$ was strongly delayed and reduced in pgr1 mutants (Figure 3B), which reflects the limited lumen acidification reported for this mutant (Jahns et al., 2002).

\section{Kinetic Correlation of Zx Synthesis and NPQ Induction}

To evaluate the kinetic correlation of NPQ induction and Zx synthesis, both parameters were plotted in the same diagram, and the maximal amplitude was scaled to the same size (Figure 4). From plots of NPQ vs. Zx (exemplarily shown in Figure S3), 


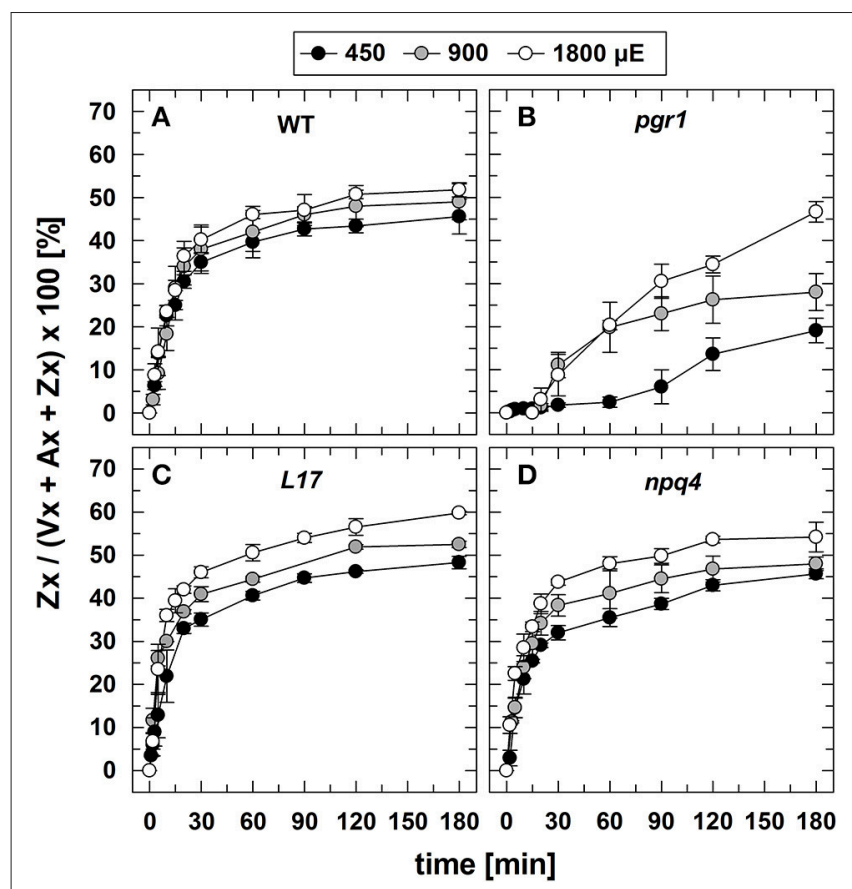

FIGURE 3 | Zx synthesis. The synthesis of Zx during 180 min of illumination at three different actinic light intensities $(450,900$, and 1,800 $\mu \mathrm{E}$ of white light) is shown for (A) WT, (B) pgr1, (C) L17, (D) npq4 plants. Detached leaves were placed on water in temperature-controlled cuvette $\left(20^{\circ} \mathrm{C}\right)$. At indicated time, leaves were rapidly frozen in liquid $\mathrm{N}_{2}$ and the pigment composition was determined by HPLC analyses. The Zx content in \% of the total VAZ-pool size (= sum of $V x, A x$ and $Z x$ ) is shown. Data represent mean values $\pm S E$ of $3-4$ independent measurements.

the respective Pearson's correlation coefficient $r$ was calculated, as indicated in each panel. For the two genotypes with an active qE quenching (WT and L17), the induction of NPQ was found to be clearly faster than $\mathrm{Zx}$ synthesis at the two lowest $\mathrm{AL}$ intensities (Figures $\mathbf{4 A - C , J - L}$, respectively), resulting in rather low correlation coefficients over the entire time range. At the lowest $\mathrm{AL}$ intensity of $450 \mu \mathrm{E}$ and in the time range from 30 to $180 \mathrm{~min}$ illumination, however, the slow increase of NPQ showed very similar kinetics than Zx synthesis in both genotypes (Figures 4A,J). At the two higher AL intensities, the correlation coefficient increased, indicating that with increasing contribution of the slowly inducible NPQ components (qZ and $\mathrm{qI}$ ) to the total NPQ, the kinetic correlation of NPQ induction and Zx synthesis is higher in these two genotypes (Figures $4 \mathrm{~B}, \mathrm{C}, \mathrm{K}, \mathrm{L}$ ). In pgr1 plants, Zx synthesis was much slower than NPQ induction throughout the whole illumination period at all $\mathrm{AL}$ intensities (Figures 4D-F), and thus yielded low correlation coefficients. Strikingly, NPQ induction and Zx synthesis were kinetically closely correlated in the full time range in $n p q 4$ plants (Figures 4G-I) at all AL intensities as obvious from the high correlation coefficients $(>0.98)$. These data indicate that $\mathrm{Zx}$ synthesis is kinetically correlated rather with the slowly developing NPQ states only.

The correlation of $\mathrm{Zx}$ synthesis with the induction of slowly developing NPQ states in the qE-active genotypes (WT and
L17) at the two highest AL intensities is depicted more detailed in Figure 5. Here, the data were scaled in a way that the zero point of the scale for $\mathrm{Zx}$ synthesis matches the NPQ scale at the end point of the $\mathrm{qE}$ induction (about 1.5 in WT plants and 3.1 in $L 17$ plants). Hence, this plot emphasizes the correlation of $\mathrm{Zx}$ induction with the slowly inducible NPQ states is shown (compare also Figures S3B,D). This analysis yielded high correlation coefficients $(>0.97)$ and clearly demonstrates that the kinetics of Zx synthesis is kinetically closely correlated with the induction of the slowly developing NPQ states. It should be noted, however, that the activation of these NPQ states does not necessarily require $\mathrm{Zx}$, since NPQ states with similar characteristics were induced in absence of $\mathrm{Zx}(n p q 1)$ and in presence of $100 \% \mathrm{Zx}$ (npq2).

\section{NPQ Relaxation Characteristics in Dependence of the Intensity and Duration of Pre-Illumination}

The relaxation of NPQ after different times of pre-illumination at the three AL intensities is summarized in Figure 6 and the detailed analysis of the NPQ relaxation kinetics characteristics is listed in Tables S1-S3 for the three AL intensities 450, 900, and $1,800 \mu \mathrm{E}$, respectively. In genotypes with an active $\mathrm{qE}$ mechanism (WT, $L 17$ and $n p q 2$ ), rapid (= within $3 \mathrm{~min}$ ) relaxation of NPQ dominated under all conditions (Figures 6A,C,F and Tables S1$\mathrm{S} 3)$. The decay time $(\tau)$ of $\mathrm{qE}$ was in the range from 10 to $50 \mathrm{~s}$ for WT and 217 plants, and in the range from 100 to $200 \mathrm{~s}$ for $n p q 2$ plants (Tables S1-S3), in agreement with earlier work (Nilkens et al., 2010). A clear additional contribution of a more slowly relaxing component was detectable in these genotypes after increasing illumination times, particularly at the highest $\mathrm{Al}$ intensity of $1,800 \mu \mathrm{E}$. This component has been assigned to the qZ component of NPQ (Nilkens et al., 2010). and its relaxation time increased with increasing illumination time at all AL intensities from 200 to $500 \mathrm{~s}$ after 5 min pre-illumination up to 2,000-5,000 s after 180 min of pre-illumination (Tables S1S3). It is important to note, that this component, which has been correlated with the epoxidation of $\mathrm{Zx}$ to $\mathrm{Vx}$ in WT plants, is present with similar characteristics also in $n p q 2$ plants, which are unable to convert $\mathrm{Zx}$ to $\mathrm{Vx}$. A third component, which was irreversible in the time range of the measurements and thus was assigned to the qI component (= photoinhibition) was detectable particularly at longer times of pre-illumination. The amplitude of this component increased with increasing illumination time and increasing AL intensities in all genotypes (Figure 6 and Tables S1-S3).

In pgr1 (Figure 6B) and $n p q 4$ (Figure 6D) mutants, which are impaired in $\mathrm{qE}$ activation, a rapidly relaxing NPQ component was missing. The most rapid component of NPQ relaxation in these two mutants matched in most cases the characteristics of the medium (qZ) component of NPQ relation observed in WT plants (Tables S1-S3). In contrast to the qZ phase in WT plants, however, this component became accelerated in pgr1 and npq4 after illumination with the highest AL intensity of 1,800 $\mu \mathrm{E}$. Both mutants further showed a more slowly relaxing component, which was characterized by very slow kinetics ( $\tau>2,000 \mathrm{~s})$ and 


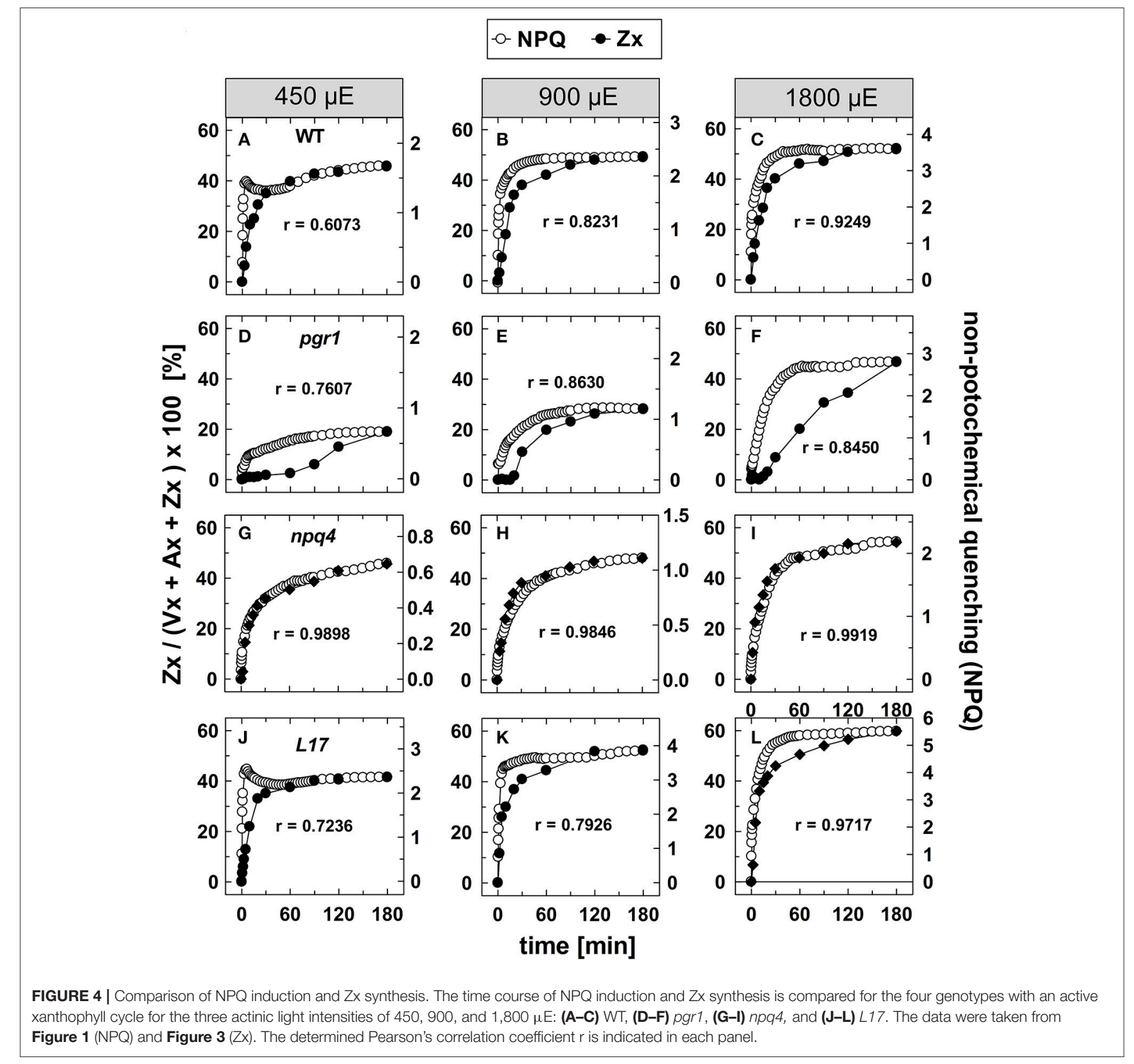

additionally, like in WT plants, an irreversible part of NPQ, which can be assigned to qI.

Unique NPQ relaxation characteristics were found for $n p q 1$ plants (Figure 6E). In this mutant, a rapidly relaxing component was detectable, though with lower amplitudes than in WT plants. The latter is related to a fraction of $\mathrm{qE}$ that is activated even in absence of $\mathrm{Zx}$ (Figure 1). It should be noted, however, that the amplitude of rapidly relaxing NPQ in $n p q 1$ was reduced in comparison to the amplitude of the rapidly induced NPQ under all conditions (Tables S1-S3). Moreover, the relaxation kinetics were clearly retarded with increasing time of pre-illumination at all AL intensities. The slowly relaxing NPQ components, however, showed similar characteristics in comparison with WT plants. Hence, NPQ relaxation similar to the $\mathrm{qZ}$ component of WT plants is present even in absence of Zx.

In conclusion, NPQ relaxation in the different genotypes differed predominantly with respect to the rapidly relaxing $\mathrm{qE}$ component, but apart from that showed very similar responses to varying light treatments. Increasing illumination time led to retardation of NPQ relaxation in all genotypes and at all AL intensities. For all times of pre-illumination, this retardation was more apparent at higher $\mathrm{AL}$ intensities, reflecting the increased induction of photoinhibitory processes under these conditions. However, the retardation of NPQ relaxation was 


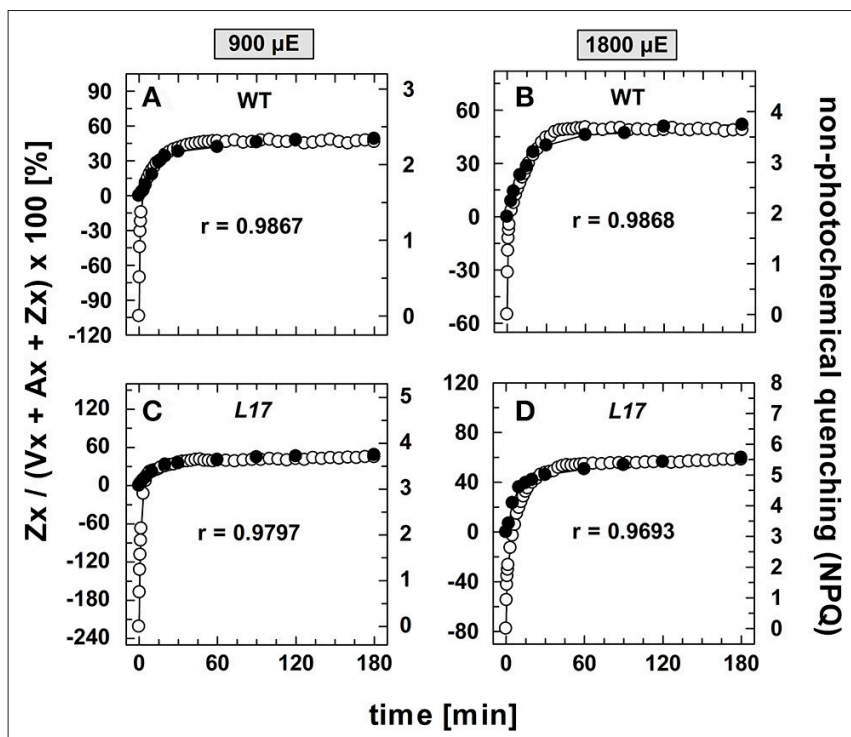

FIGURE 5 | Comparison of the kinetics of NPQ induction and Zx synthesis in WT and $L 17$ plants. The data for NPQ induction (open circles) and Zx synthesis (filled circles) are compared for WT plants (A,B) and $L 17$ plants (C,D) at 900, (A,C) and 1,800 $\mu \mathrm{E}$ (B,D). For direct comparison, the data for Zx synthesis were fitted to match the amplitudes of the slowly developing (> 2 min) NPQ components, only. The data were taken from Figure 1 (NPQ) and Figure $\mathbf{3}(Z \mathrm{x})$. The determined Pearson's correlation coefficient $r$ is indicated in each panel.

less pronounced in genotypes with an active $\mathrm{qE}$ mechanism, namely WT (Figure 6A), L17 (Figure 6C), and npq2 (Figure 6F), in comparison to those with an impaired qE quenching, pgr1 (Figure 6B), $n p q 4$ (Figure 6D) and $n p q 1$ (Figure 6E), indicating a photoprotective role of $\mathrm{qE}$ quenching. However, the maximum absolute levels of slowly relaxing NPQ components, i.e., the amplitude of $\mathrm{qZ}$ and $\mathrm{qI}$, was similar in most genotypes, varying from about 1.5 to 2.0. Only pgr1 mutant plants showed an increased level of slowly relaxing NPQ with an amplitude of about 2.5-3.0.

\section{Zx Epoxidation Characteristics}

The reconversion of $\mathrm{Zx}$ to $\mathrm{Vx}$, i.e., the epoxidation of $\mathrm{Zx}$, was determined under the same conditions for the four genotypes with an active xanthophyll cycle (Figure S2). For a better comparison of the epoxidation kinetics, the data are depicted in a normalized way in Figure 7. For Zx epoxidation, no detailed kinetics analysis was performed for two reasons: First, due to the rather low amount of data points the amplitudes and kinetics could not be fitted with sufficient reliability. Second, Zx epoxidation does not follow exponential decay characteristics, but represents a two-stepped consecutive reaction, which further limits accurate fitting with few data points. The reconversion of $\mathrm{Zx}$ to $\mathrm{Vx}$ showed in general the same features as the NPQ relaxation, except for the absence of a rapid phase with decay times in the range from 1 to $2 \mathrm{~min}$. However, similar to the two slower NPQ relaxation components, epoxidation of $\mathrm{Zx}$ was retarded in response to increasing illumination time and increasing $\mathrm{AL}$ intensity for all genotypes (Figure 7). For pgr1 plants, epoxidation was only determined for the two longest times of pre-illumination (90 and $180 \mathrm{~min}$ ), because only very low levels of $\mathrm{Zx}$ were formed at shorter illumination times of 15 and $30 \mathrm{~min}$ (Figure 3).

\section{Kinetic Correlation of Zx Epoxidation and NPQ Relaxation}

To compare the kinetics of NPQ relaxation and Zx epoxidation, both data sets were plotted together for each time or preillumination and for all $\mathrm{AL}$ intensities upon normalization to the same initial amplitudes. This comparison is shown exemplarily for the data obtained after $90 \mathrm{~min}$ of pre-illumination in Figure 8, while the data for the three other conditions $(15,30$, and $180 \mathrm{~min}$ of pre-illumination) are summarized in Figures S4-S6, respectively. Again, the respective Pearson's correlations coefficients derived from separate plots of NPQ vs $\mathrm{Zx}$, are indicated in each panel. It is clearly visible, that the epoxidation of $\mathrm{Zx}$ occurred with slower kinetics than NPQ relaxation in the two qE-active genotypes, WT and L17 (Figures 8A-C,J-L, respectively), and that particularly the rapid relaxation of the $\mathrm{pH}$-dependent $\mathrm{qE}$ component of NPQ was not paralleled by $\mathrm{Zx}$ epoxidation (correlation coefficients between about 0.8 and 0.9 ). In contrast, $\mathrm{Zx}$ epoxidation in the qE-deficient $n p q 4$ mutant followed quite similar kinetics than NPQ relaxation (Figures 8G-I) and thus yielded high correlation coefficients of about 0.98 . In pgr1 plants, however, Zx epoxidation kinetics matched NPQ relaxation kinetics after preillumination at the lowest AL intensity of $450 \mu \mathrm{E}$ (Figure 8D), while $\mathrm{Zx}$ reconversion was more retarded than NPQ relaxation at increasing AL intensities (Figures 8E,F). The same general trends were observed for other times of pre-illumination (Figures S4S6). These analyses indicate that $\mathrm{Zx}$ epoxidation is kinetically correlated with the $\mathrm{pH}$-independent, slowly relaxing NPQ components, which have been assigned in WT plants to qZ and qI (Nilkens et al., 2010). For a better visualization of this correlation, the amplitudes of $\mathrm{Zx}$ epoxidation data were fitted to match the amplitudes of the more slowly relaxing NPQ components, $\mathrm{qZ}$ and $\mathrm{qI}$, only. This analysis is again shown exemplarily for the data determined after 90 min of pre-illumination (Figure 9), while the data for 5, 30, and $180 \mathrm{~min}$ of pre-illumination are given in Figures S7-S9, respectively. When applying this way of analysis, the epoxidation of $\mathrm{Zx}$ matched nearly perfectly the kinetics of the slower, $\mathrm{pH}$-independent NPQ components in the two qE-active genotypes, WT (Figures 9A-C) and L17 (Figures 9J-L), as reflected by the high correlation coefficients. Note, that the dashed lines in the panels for these two genotypes indicate the NPQ amplitudes after relaxation of qE. In contrast, Zx epoxidation and NPQ relaxation were closely correlated for the entire time frame of the measurements in npq4 mutants at all three AL intensities (Figure 9G-I), and for the two lowest AL intensities also in pgr1 plants (Figures 9D,E). In the latter genotype, the correlation was restricted to the slowest phase of NPQ relaxation only at the highest AL intensity of $1,800 \mu \mathrm{E}$ (Figure 9F). The same characteristics were observable for all other times of pre-illumination (Figures S7-S9). In conclusion, 


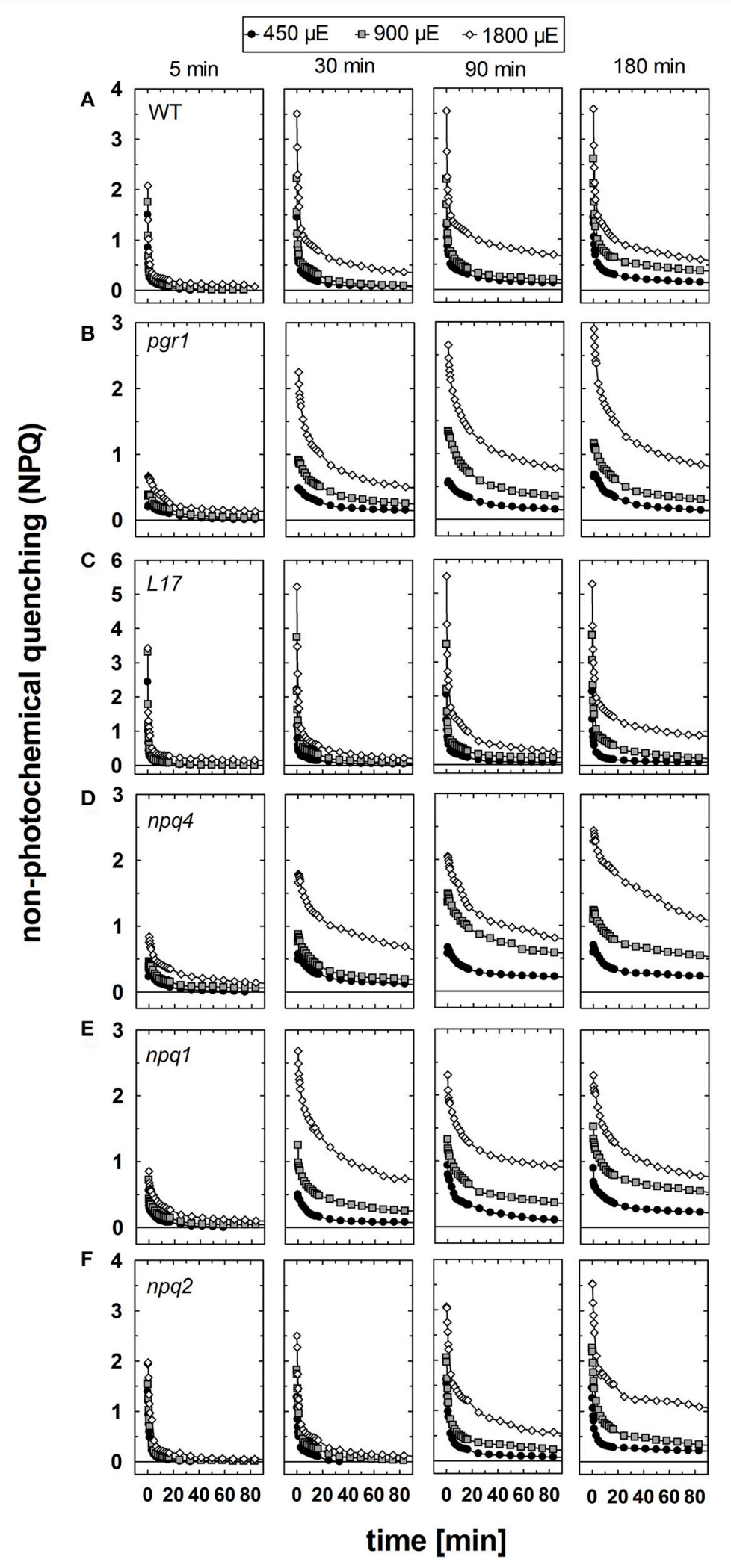

FIGURE 6 | NPQ relaxation. The dark relaxation of NPQ after pre-illumination at three different actinic light intensities (450, 900, and 1,800 $\mu \mathrm{E}$ of white light) for 5, 30, 90, and 180 min was determined for (A) WT, (B) pgr1, (C) L17, (D) npq4, (E), npq2, and (F) npq1 plants. During the whole measurements, detached leaves were placed on wet paper in a temperature-controlled cuvette $\left(20^{\circ} \mathrm{C}\right)$ under permanent supply with ambient air. Mean values of $3-6$ independent measurements are shown. For clarity, error bars, which ranged from 0.1 to 0.3 in all cases, are not shown. 


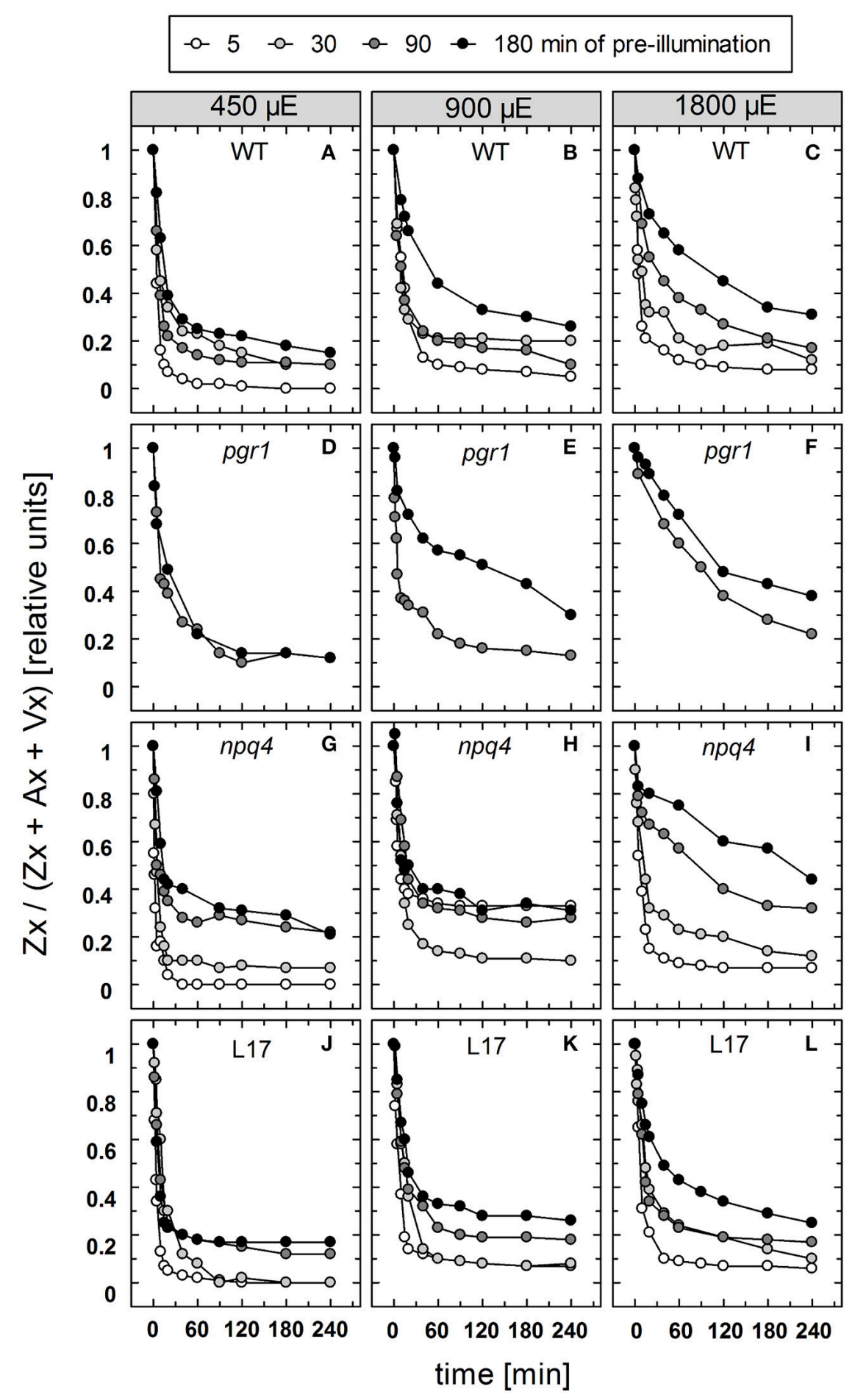

FIGURE 7 | Zx epoxidation. The reconversion of Zx to $V x$ after pre-illumination at three different actinic light intensities (450, 900, and 1,800 $\mu E$ of white light) for 5 , 30, 90, and 180 min was determined for WT (A-C), pgr1 (D-F), npq4 (G-I), and L17 (J-L) plants. During the whole experiment, detached leaves were placed on water in temperature-controlled cuvette $\left(20^{\circ} \mathrm{C}\right)$. At indicated time, leaves were rapidly frozen in liquid $\mathrm{N}_{2}$ and the pigment composition was determined by $\mathrm{HPLC}$ analyses. The Zx content in \% of the total VAZ-pool size (= sum of Vx, Ax and Zx) is shown. Data represent mean values of 3-4 independent measurements. For clarity, error bars, which ranged from 1 to $5 \%$ in all cases, are not shown. 


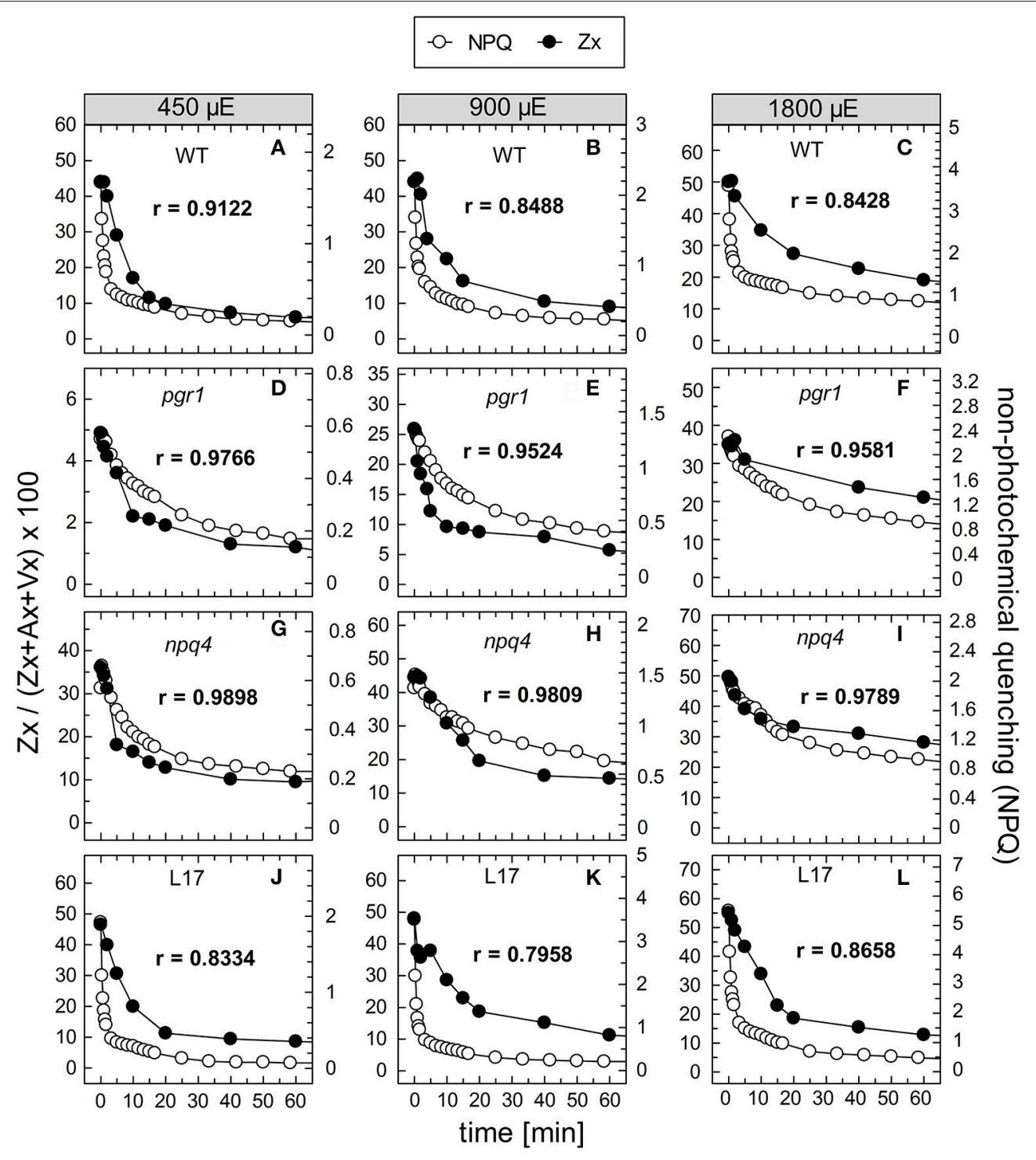

FIGURE 8 | Comparison of NPQ relaxation and Zx epoxidation after 90 min of pre-illumination. The time course of NPQ relaxation and Zx epoxidation after 90 min of pre-illumination at the three actinic light intensities of 450,900 , and $1800 \mu \mathrm{E}$ is compared for the four genotypes with an active xanthophyll cycle: (A-C) WT, (D-F) pgr1, (G-I) npq4, and (J-L) L17. The data were taken from Figure $\mathbf{6}(\mathrm{NPQ})$ and Figure $\mathbf{7}(\mathrm{Zx})$. The determined Pearson's correlation coefficient $r$ is indicated in each panel.

these analyses provide strong evidence that the kinetics of $\mathrm{Zx}$ epoxidation are closely correlated with the kinetics of slowly relaxing NPQ components in all genotypes with an active xanthophyll cycle.

\section{DISCUSSION}

The close kinetic correlation of $\mathrm{Zx}$ conversion and the dynamics of slowly inducible/relaxing NPQ states (qZ and qI) indicate an essential photoprotective function of $\mathrm{Zx}$ in these processes. While such a function has been proposed earlier, it remains a matter of debate, whether $\mathrm{Zx}$ is directly involved in the underlying quenching processes or whether it serves indirect functions as allosteric modulator of quenching processes or general photoprotective functions in the lipid phase of the membrane. Though the simple kinetical correlation cannot provide an answer to this question, the observed NPQ characteristics in different mutants support the view, that the slowly developing NPQ states can be induced independent of xanthophyll conversion. Such an interpretation provides a strong argument against a direct role of $\mathrm{Zx}$ in $\mathrm{qZ}$ and $\mathrm{qI}$. This hypothesis is discussed in the following in context with the current knowledge of the literature related to a direct or indirect function of $\mathrm{Zx}$ in NPQ. It should further be noted, that the slowly inducible NPQ states are not related to state transitions $(\mathrm{qT})$ or chloroplast movement. The contribution of 


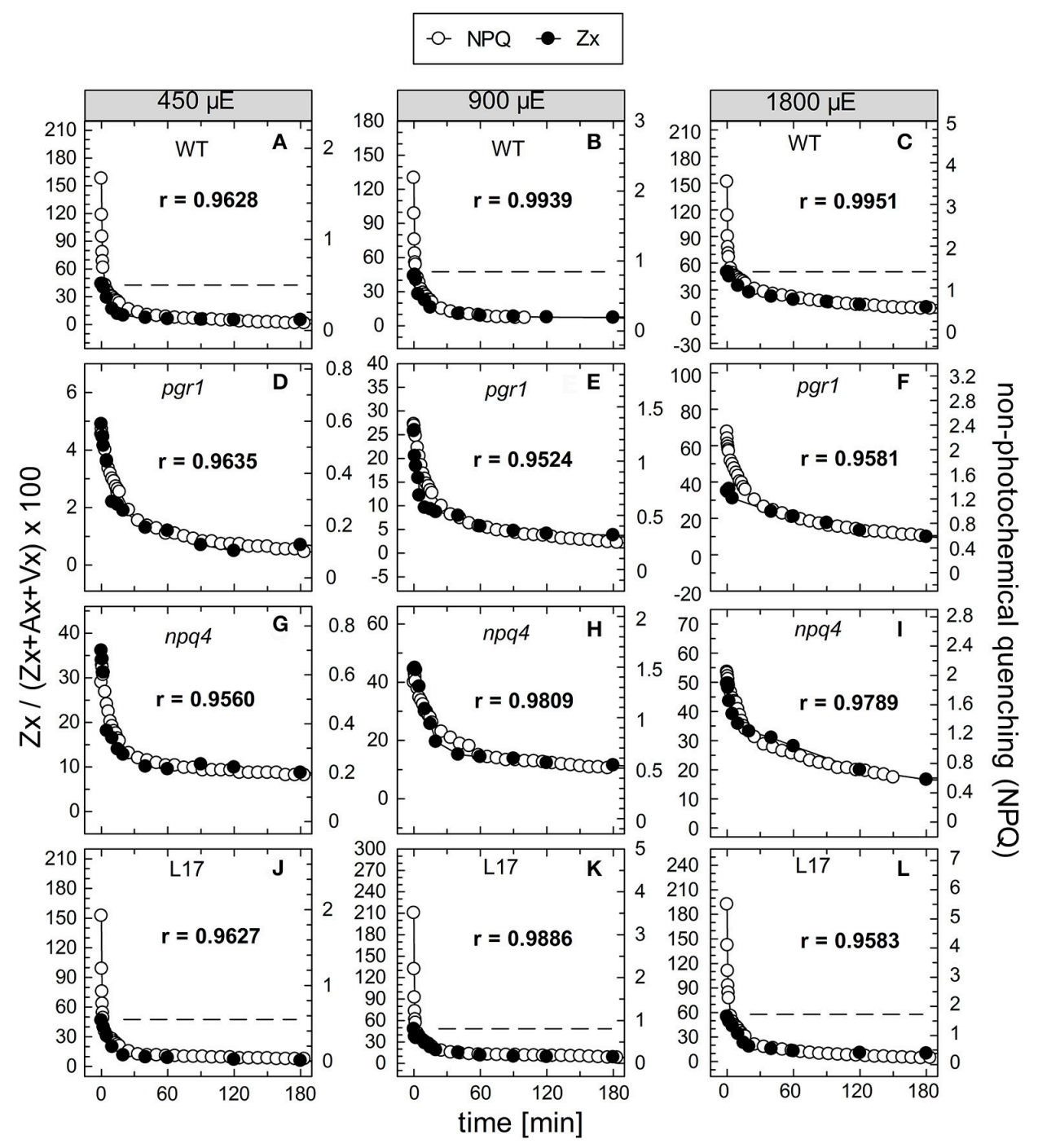

FIGURE 9 | Comparison of the kinetics of NPQ relaxation and Zx epoxidation after 90 min of pre-illumination. The data for NPQ relaxation (open circles) and Zx epoxidation (filled circles) after 90 min of pre-illumination at the three actinic light intensities of 450, 900, and 1,800 $\mu \mathrm{E}$ are compared for the four genotypes with an active xanthophyll cycle: (A-C) WT, (D-F) pgr1, (G-I) npq4 and (J-L) L17. For direct comparison, the data for Zx epoxidation were fitted to match the amplitudes of the slowly relaxing (> $2 \mathrm{~min}) \mathrm{NPQ}$ components, only. The data were taken from Figure $\mathbf{6}(\mathrm{NPQ})$ and Figure $\mathbf{7}(\mathrm{Zx})$. The dotted lines in panels (A-C) and (J-L) indicate the NPQ amplitudes after relaxation of qE. The determined Pearson's correlation coefficient $r$ is indicated in each panel.

qT to NPQ under these experimental conditions can be excluded from earlier work with the qT-deficient stn 7 mutant, which showed similar NPQ dynamics than WT plants under the same conditions (Nilkens et al., 2010). The possible contribution of chloroplast movement, which is triggered by blue light, was excluded from the comparison of the NPQ induction by either white (= blue and red light) or red actinic light (Figure S10). In both cases, very similar NPQ induction dynamics were determined.

\section{Quenching Mechanisms and Quenching Sites}

Any direct function of $\mathrm{Zx}$ in NPQ requires a specific binding site for the deactivation of excited Chl molecules. Because xanthophylls bind to $\mathrm{Chl} \mathrm{a} / \mathrm{b}$ antenna proteins only, direct quenching by $\mathrm{Zx}$ can be expected to occur exclusively in antenna proteins, but not in reaction center proteins. So far, a direct function of $\mathrm{Zx}$ has been proposed only for the $\mathrm{pH}$ regulated $\mathrm{qE}$ mechanism. Experimental evidence for a direct $\mathrm{Zx}$ function, related to a charge transfer state, which involves electron transfer from $\mathrm{Zx}$ to $\mathrm{Chl}$, was derived from transient absorption measurements in the near infrared region (Holt et al., 2005; Ahn et al., 2008; Avenson et al., 2008, 2009; Dall'Osto et al., 2017). This mechanism was described to be active in isolated thylakoid membranes (Holt et al., 2005) and in isolated minor PSII antenna complexes Lhcb4-6 (Ahn et al., 2008; Avenson et al., 2008), but not in trimeric LHCII (Avenson et al., 2008). From recent analysis of the subunit 
and $\mathrm{Chl}$ organization in the PSII $\mathrm{C}_{2} \mathrm{~S}_{2} \mathrm{M}_{2}$ supercomplex from Arabidopsis it was concluded, that Lhcb5 (CP26), Lhcb4 (CP29), and LHCII S-trimers can transfer excitation energy directly to the reaction center core (CP43), while LHCII M-trimers transfer excitation energy either through S-trimers or Lhcb4 (CP29) (van Bezouwen et al., 2017). This suggests that energy flow from the antenna to the reaction center occurs mainly through S-trimers or Lhcb4, but not through Lhcb5, which makes Lhcb5 a much less favorable candidate for efficient quenching compared to Lhcb4. Recent analysis of mutants devoid of minor antenna complexes provided evidence that two mechanisms of $\mathrm{qE}$ quenching exist: one in minor antenna complexes, which involves the formation of a xanthophyll cation radical and another in trimeric LHCII, which does not involve a charge transfer state but requires Zx (Dall'Osto et al., 2017). Although it remains to be clarified, to what extent these characterized charge transfer states contribute to $\mathrm{qE}$ under in vivo conditions in the fully assembled PSII antenna, these studies indicate a dual role of $\mathrm{Zx}$ in energy dissipation, combining a direct and indirect function.

In contrast, an exclusively indirect function of $\mathrm{Zx}$ in $\mathrm{qE}$ is favored by Horton, Ruban and co-workers, who suggest that $\mathrm{Zx}$ mainly modulates the efficiency of $\mathrm{qE}$ by shifting the $\mathrm{pH}$-dependence of $\mathrm{qE}$ to higher $\mathrm{pH}$ values (Horton et al., 2000). In their model, energy dissipation in antenna proteins is facilitated by energy transfer to lutein (Ruban et al., 2007), and activation of quenching is essentially triggered by $\mathrm{pH}$ regulated conformational changes in PSII antenna proteins (for a review see Ruban et al., 2012). Finally, a carotenoid independent quenching mechanism based on the formation of a Chl-Chl charge transfer state has been postulated as mechanism for $\mathrm{qE}$ quenching on basis of $\mathrm{Chl}$ fluorescence and transient absorption spectroscopy (Miloslavina et al., 2008; Muller et al., 2010). Based on simulations of the molecular dynamics of monomeric PSII antenna proteins, it has recently been proposed that $\mathrm{Zx}$ rather supports conformational changes at the luminal side of antenna proteins without direct contribution to NPQ (Papadatos et al., 2017).

Hence, all different approaches support an indirect role of $\mathrm{Zx}$ in $\mathrm{qE}$, while an additional direct function was proposed on basis of transient absorption spectroscopy. It should be noted, however, that all of these studies are limited by the fact that no intact leaves have been used as material. This is an important issue, since NPQ activation is a complex property of the entire chloroplast, and activation of $\mathrm{qE}$ involves not only structural reorganization of PSII complexes but also of the thylakoid membrane (Kirchhoff, 2014; Demmig-Adams et al., 2015; Schumann et al., 2017). The structural properties and NPQ characteristics of isolated thylakoid membranes and recombinant antenna proteins can be expected to differ from that of intact chloroplasts. Applying time-resolved fluorescence analysis to intact, detached Arabidopsis leaves, Holzwarth and co-workers identified two different quenching sites that were activated under in vivo conditions along with NPQ induction (Holzwarth et al., 2009). One site was found to depend on the presence of $\mathrm{Zx}$ and was localized to antenna proteins associated with PSII reactions center. It remained unclear, however, whether $\mathrm{Zx}$ acts as direct quencher of energy or simply as regulator of a $\mathrm{Zx}$-independent quenching process and whether the $\mathrm{Zx}$-dependent quenching site contributes to $\mathrm{qE}$ or to $\mathrm{qZ}$.

For the two slowly inducible and relaxing NPQ components, $\mathrm{qZ}$ and $\mathrm{qI}$, no detailed mechanisms have been proposed so far. For qZ, the quenching has been assigned to processes involving the minor antenna protein Lhcb5 (Dall'Osto et al., 2005), but it remains to be elucidated, whether also other antenna proteins contribute to $\mathrm{qZ}$. As pointed out above, however, analysis of the energy transfer pathways in PSII-LHCII supercomplexes do not favor energy quenching in Lhcb5 (van Bezouwen et al., 2017). Moreover, the exact role of $\mathrm{Zx}$ in $\mathrm{qZ}$ is still unclear. The induction of NPQ states with similar characteristics as $\mathrm{qZ}$ in the two xanthophyll cycle mutants $n p q 1$ and $n p q 2$ (Figures 1, 6) argue against any direct function of $\mathrm{Zx}$ in $\mathrm{qZ}$. The photoinhibitory quenching $\mathrm{qI}$ is based on the high-light induced damage of the reaction center protein D1, but the underlying molecular quenching mechanism is unknown. A direct role $\mathrm{Zx}$ in $\mathrm{qI}$ seems very unlikely, since $\mathrm{Zx}$ binding to PSII reaction center proteins has not been observed. The close correlation of the relaxation of $\mathrm{qZ}$ and $\mathrm{qI}$ with $\mathrm{Zx}$ epoxidation thus strongly supports an indirect role $\mathrm{Zx}$ in $\mathrm{qZ}$ and $\mathrm{qI}$.

\section{Zx Binding Sites and Xanthophyll Conversion Characteristics}

A direct function of $\mathrm{Zx}$ in energy dissipation involving a Chl-Zx charge transfer state has been assigned to Zx located at the L2 site (Holt et al., 2005; Ahn et al., 2008; Avenson et al., 2008), while quenching by lutein has been proposed to occur at the L1 site (Ruban et al., 2007; Avenson et al., 2009). Thus, a direct function of $\mathrm{Zx}$ essentially requires binding to the L2 site in minor antenna proteins. The light-induced exchange of $\mathrm{Vx}$ by $\mathrm{Zx}$ at the L2 site is therefore a prerequisite of a direct quenching function of Zx. Binding of $\mathrm{Vx}$ to the L2 site has been reported for Lhcb6 (Passarini et al., 2009) and Lhcb4 (Pan et al., 2011; Wei et al., 2016). In Lhcb5, this binding site is predominantly occupied by Lut (Wei et al., 2016), but may also be occupied by Vx (Ballottari et al., 2009). It is under debate, however, to what extent $\mathrm{Vx}$ bound at the $\mathrm{L} 2$ site is convertible to $\mathrm{Zx}$ and whether the formed $\mathrm{Zx}$ rebinds to the $\mathrm{L} 2$ site. It is known from in vitro studies that de-epoxidation of $\mathrm{Vx}$ bound to the L2 site of the minor complexes Lhcb4-6 occurs to a rather low extent (about $10-40 \%)$ only, and follows slower kinetics than at the V1 site of trimeric LHCII proteins (Morosinotto et al., 2003; Wehner et al., 2004). Consequently, only a fraction of minor complexes can be expected to bind $\mathrm{Zx}$ upon activation of $\mathrm{qE}$ and $\mathrm{qZ}$. It is further questionable, whether the formed $\mathrm{Zx}$ indeed rebinds to the L2 site. Recent comparative analysis of antenna complexes isolated from plants in the NPQ active and NPQ inactive state did not provide any evidence for $\mathrm{Zx}$ binding to $\mathrm{L} 2$ (Xu et al., 2015), which argues against an exchange of Vx by Zx at the L2 site. In conclusion, binding of $\mathrm{Zx}$ to the minor antenna complexes Lhcb4-6 at L2 is rather unlikely and occurs, if at all, only to very low extent. This view is further supported by the general 
characteristics of xanthophyll conversion. The de-epoxidation of $\mathrm{Vx}$ to Zx occurs in the lipid phase of the thylakoid membrane (Jahns et al., 2009) and does not require a specific interaction of the lumen-localized $\mathrm{VxDE}$, which catalyzes this reaction, with antenna proteins. The latter has been derived from the notion that $\mathrm{Vx}$ conversion in isolated Arabidopsis thylakoids can be catalyzed from both sides of the membrane with similar kinetics and efficiency (Macko et al., 2002). Based on this work, it was postulated that the release and diffusion of $\mathrm{Vx}$ in the lipid phase is the rate-limiting step of $\mathrm{Vx}$ conversion. Interestingly, conversion of $\mathrm{Vx}$ to $\mathrm{Zx}$ from the stroma side has recently been shown to be active under in vivo conditions in the green alga Chlamydomonas reinhardtii ( $\mathrm{Li}$ et al., 2016). In this case, the Vx converting enzyme is exclusively located in the chloroplast stroma, supporting the view that diffusion of $\mathrm{Vx}$ in the lipid phase precedes its conversion. Conversion of $\mathrm{Vx}$ in the lipid phase requires initially the release of $\mathrm{Vx}$ from the respective binding site, explaining why $\mathrm{Vx}$ bound to the peripheral V1 site occurs very rapidly and with high efficiency (Jahns et al., 2001). The release of Vx from the inner L2 site in minor PSII antenna proteins thus likely requires a pronounced structural reorganization, and the same applies to any specific rebinding of the formed $\mathrm{Zx}$ to the L2 site. The latter is complicated by the different properties of $\mathrm{Vx}$ and $\mathrm{Zx}$ related to structure and hydrophobicity. This makes it much more likely that $\mathrm{Zx}$ acts either in the membrane or at the periphery of antenna complexes rather than being rebound at very specific inner xanthophyll binding sites like L2.

\section{The Role of Non-protein Bound Zx}

A general function of $\mathrm{Zx}$ in photoprotection independent of its role in energy dissipation has been derived from studies of different Arabidopsis mutants (Havaux and Niyogi, 1999; Havaux et al., 2007; Dall'Osto et al., 2010). Such a function is likely related to the antioxidative capacity of $\mathrm{Zx}$ as nonprotein bound xanthophyll in the lipid phase of the membrane (Havaux et al., 2007; Dall'Osto et al., 2010). The amount of the xanthophyll cycle pigments (VAZ pool size) as well as the portion of photoconvertible $\mathrm{Vx}$ is known to increase upon acclimation to high light intensities in parallel with an increased NPQ capacity (Adams and Demmig-Adams, 1992; Demmig-Adams and Adams, 1992; Bailey et al., 2004; Demmig-Adams et al., 2006; Schumann et al., 2017). Because the number of antenna proteins and thus xanthophyll binding sites is reduced upon high light acclimation, the additional VAZ pigments must reside in the lipid phase of the membrane. This underlines the important role of non-protein bound $\mathrm{Zx}$ in photoprotection upon longterm high light stress. It is further known that the loss of $\mathrm{Zx}$ correlates with increased levels of tocopherols and vice versa (Havaux et al., 2000, 2005; Triantaphylides and Havaux, 2009), suggesting a central role of $\mathrm{Zx}$ in the protection against lipid peroxidation. Moreover, non-protein bound Zx may serve in the regulation of membrane properties such as fluidity or stability (Tardy and Havaux, 1997; Havaux, 1998). The close correlation of Zx levels and photoinhibitory quenching found here supports the earlier observation that ZEP activity is down-regulated concomitant with PSII activity under prolonged high light stress
(Reinhold et al., 2008) and might thus reflect the necessity to retain $\mathrm{Zx}$ in the lipid phase for photoprotection of inactivated PSII.

Up to $3 \mathrm{~h}$ illumination at the highest light intensity of 1,800 $\mu \mathrm{E}$ used here, no pronounced impact of different $\mathrm{Zx}$ levels on the slowly inducible NPQ components ( $\mathrm{qZ}$ and $\mathrm{qI}$ ) was observable (Figures 1, 2). This implies that the inactivation of PSII occurs independent of the presence of $\mathrm{Zx}$, suggesting a photoprotective role of $\mathrm{Zx}$ in longer lasting high light stress, as has been shown for the Zx-deficient npq1 mutant (Havaux and Niyogi, 1999; Havaux et al., 2000). The gradual downregulation of $\mathrm{Zx}$ epoxidation in parallel with the recovery from photoinhibition thus rather reflects a protective role of $\mathrm{Zx}$ during PSII repair cycle than a direct function of Zx in qI quenching. The coordinated down-regulation of both Zx epoxidation and NPQ relaxation upon increasing high-light stress requires a strict coregulation of both processes. So far, the molecular mechanism of the down-regulation of ZEP activity is unknown. However, recent work showed that mutants with deficient NADPH thioredoxin reductase $\mathrm{C}$ (NTRC) accumulate higher levels of Zx, indicating a partial inactivation of ZEP in absence of thioredoxin reduction (Naranjo et al., 2016). This supports a crucial role of thioredoxin mediated reduction of disulphides in the regulation of ZEP activity and suggests that oxidation of cysteine residues might be responsible for ZEP inactivation under prolonged high light stress. The retainment of high $\mathrm{Zx}$ levels along with the repair of damaged PSII seems to be central for protecting PSII centers during the repair cycle. Related to such a role, the $\mathrm{Zx}$ content and the $\mathrm{Zx}$ dynamics thus represent a molecular memory of experienced high light stress. From the physiological point of view, this will allow not only photoprotection of inhibited PSII centers, but likely also a more rapid or efficient reactivation of NPQ states after recurrent high light stress.

In conclusion, the comparison of the dynamics of NPQ and $\mathrm{Zx}$ levels strongly support the hypothesis that $\mathrm{Zx}$ has no direct function in the quenching mechanisms related to the two slowly relaxing NPQ components qZ and qI. This interpretation is derived from the similar characteristics of these two NPQ components independent of the different $\mathrm{Zx}$ levels in the studied genotypes. The observed slight differences in the NPQ development in the different mutants as compared to WT plants might be either due to differences in the $\mathrm{qE}$ properties or might reflect alterations in the modulation of PSII downregulation in dependence of $\mathrm{Zx}$, as has been shown also for $\mathrm{qE}$. Hence, Zx has likely an allosteric function in the modulation of slowly inducible NPQ states, which might additionally involve general photoprotective functions in the lipid phase of the membrane.

\section{AUTHOR CONTRIBUTIONS}

PJ: Planned and designed the experiments; EK: Performed the experiments; Both authors analyzed and interpreted the data. PJ wrote the MS. Both authors read and approved the final version of the manuscript. 


\section{FUNDING}

This work was supported by the Deutsche Forschungsgemeinschaft (DFG) to PJ (JA 665/8-1 and GRK 1525).

\section{REFERENCES}

Adams, W. W., and Demmig-Adams, B. (1992). Operation of the xanthophyll cycle in higher plants in response to diurnal changes in incident sunlight. Planta 186, 390-398. doi: 10.1007/BF00195320

Adams, W. W., Demmig-Adams, B., Rosenstiel, T. N., Brightwell, A. K., and Ebbert, V. (2002). Photosynthesis and photoprotection in overwintering plants. Plant Biol. 4, 545-557. doi: 10.1055/s-2002-35434

Ahn, T. K., Avenson, T. J., Ballottari, M., Cheng, Y. C., Niyogi, K. K., Bassi, R., et al. (2008). Architecture of a charge-transfer state regulating light harvesting in a plant antenna protein. Science 320, 794-797. doi: 10.1126/science.1154800

Allen, J. F. (2003). State transitions - a question of balance. Science 299, 1530-1532. doi: $10.1126 /$ science. 1082833

Allen, J. F., Bennett, J., Steinback, K. E., and Arntzen, C. J. (1981). Chloroplast protein phosphorylation couples plastoquinone redox state to distribution of excitation energy between photosystems. Nature 291, 25-29. doi: $10.1038 / 291025 \mathrm{a} 0$

Armbruster, U., Carrillo, L. R., Venema, K., Pavlovic, L., Schmidtmann, E., Kornfeld, A., et al. (2014). Ion antiport accelerates photosynthetic acclimation in fluctuating light environments. Nat. Commun. 5:5439. doi: $10.1038 /$ ncomms6439

Aro, E. M., Virgin, I., and Andersson, B. (1993). Photoinhibition of photosystem, II. Inactivation, protein damage and turnover. Biochim. Biophys. Acta 1143, 113-134. doi: 10.1016/0005-2728(93)90134-2

Avenson, T. J., Ahn, T. K., Niyogi, K. K., Ballottari, M., Bassi, R., and Fleming, G. R. (2009). Lutein can act as a switchable charge transfer quencher in the CP26 light-harvesting complex. J. Biol. Chem. 284, 2830-2835. doi: 10.1074/jbc.M807192200

Avenson, T. J., Ahn, T. K., Zigmantas, D., Niyogi, K. K., Li, Z., Ballottari, M., et al. (2008). Zeaxanthin radical cation formation in minor lightharvesting complexes of higher plant antenna. J. Biol. Chem. 283, 3550-3558. doi: 10.1074/jbc.M705645200

Bailey, S., Horton, P., and Walters, R. G. (2004). Acclimation of Arabidopsis thaliana to the light environment: the relationship between photosynthetic function and chloroplast composition. Planta 218, 793-802. doi: 10.1007/s00425-003-1158-5

Ballottari, M., Mozzo, M., Croce, R., Morosinotto, T., and Bassi, R. (2009). Occupancy and functional architecture of the pigment binding sites of photosystem II antenna complex Lhcb5. J. Biol. Chem. 284, 8103-8113. doi: 10.1074/jbc.M808326200

Bellafiore, S., Bameche, F., Peltier, G., and Rochaix, J. D. (2005). State transitions and light adaptation require chloroplast thylakoid protein kinase STN7. Nature 433, 892-895. doi: 10.1038/nature03286

Bonardi, V., Pesaresi, P., Becker, T., Schleiff, E., Wagner, R., Pfannschmidt, T., et al. (2005). Photosystem II core phosphorylation and photosynthetic acclimation require two different protein kinases. Nature 437, 1179-1182. doi: 10.1038/nature04016

Briantais, J.-M., Vernotte, C., Picaud, M., and Krause, G. H. (1979). A quantative study of the slow decline of chlorophyll $a$ fluorescence in isolated chloroplasts. Biochim. Biophys. Acta 548, 128-138. doi: 10.1016/0005-2728(79)90193-2

Correa-Galvis, V., Poschmann, G., Melzer, M., Stühler, K., and Jahns, P. (2016). PsbS interactions involved in the activation of energy dissipation in Arabidopsis. Nat. Plants 2:15225. doi: 10.1038/nplants.2015.225

Dall'Osto, L., Caffarri, S., and Bassi, R. (2005). A mechanism of nonphotochemical energy dissipation, independent from PsbS, revealed by a conformational change in the antenna protein CP26. Plant Cell 17, 1217-1232. doi: 10.1105/tpc.104.030601

Dall'Osto, L., Cazzaniga, S., Bressan, M., Palecek, D., Zidek, K., Niyogi, K. K., et al. (2017). Two mechanisms for dissipation of excess light in

\section{SUPPLEMENTARY MATERIAL}

The Supplementary Material for this article can be found online at: https://www.frontiersin.org/articles/10.3389/fpls.2017. 02094/full\#supplementary-material

monomeric and trimeric light-harvesting complexes. Nat. Plants 3:17033. doi: $10.1038 /$ nplants.2017.33

Dall'Osto, L., Cazzaniga, S., Havaux, M., and Bassi, R. (2010). Enhanced photoprotection by protein-bound vs free xanthophyll pools: a comparative analysis of chlorophyll b and xanthophyll biosynthesis mutants. Mol. Plant 3, 576-593. doi: 10.1093/mp/ssp117

Demmig, B., Winter, K., Krüger, A., and Czygan, F.-C. (1987). Photoinhibition and zeaxanthin formation in intact leaves. A possible role of the xanthophyll cycle in the dissipation of excess light. Plant Physiol. 84, 218-224. doi: $10.1104 /$ pp.84.2.218

Demmig-Adams, B. (1990). Carotenoids and photoprotection in plants: a role for the xanthophyll zeaxanthin. Biochim. Biophys. Acta 1020, 1-24. doi: 10.1016/0005-2728(90)90088-L

Demmig-Adams, B., and Adams, W. W. (1992). Carotenoid composition in sun and shade leaves of plants with different life forms. Plant Cell Environ. 15, 411-419. doi: 10.1111/j.1365-3040.1992.tb00991.x

Demmig-Adams, B., and Adams, W. W. (1996). The role of xanthophyll cycle carotenoids in the protection of photosynthesis. Trends Plant Sci. 1, 21-26. doi: 10.1016/S1360-1385(96)80019-7

Demmig-Adams, B., Cohu, C. M., Muller, O., and Adams, W. W. III (2012). Modulation of photosynthetic energy conversion efficiency in nature: from seconds to seasons. Photosynth. Res. 113, 75-88. doi: 10.1007/s11120-012-9761-6

Demmig-Adams, B., Ebbert, V., Mellman, D. L., Mueh, K. E., Schaffer, L., Funk, C., et al. (2006). Modulation of PsbS and flexible vs sustained energy dissipation by light environment in different species. Physiol. Plant. 127, 670-680. doi: 10.1111/j.1399-3054.2006.00698.x

Demmig-Adams, B., Muller, O., Stewart, J. J., Cohu, C. M., and Adams, W. W. (2015). Chloroplast thylakoid structure in evergreen leaves employing strong thermal energy dissipation. J. Photochem. Photobiol. B Biol. 152, 357-366. doi: 10.1016/j.jphotobiol.2015.03.014

Ebbert, V., Adams, W. W., Mattoo, A. K., Sokolenko, A., and Demmig-Adams, B. (2005). Up-regulation of a photosystem II core protein phosphatase inhibitor and sustained D1 phosphorylation in zeaxanthin-retaining, photoinhibited needles of overwintering Douglas fir. Plant Cell Environ. 28, 232-240. doi: 10.1111/j.1365-3040.2004.01267.x

Ebbert, V., Demmig-Adams, B., Adams, W. W. I., Mueh, K. E., and Staehelin, L. A. (2001). Correlation between persistent forms of zexanthin-dependent energy dissipation and thylakoid protein phosphorylation. Photosynth. Res. 67, 63-78. doi: 10.1023/A:1010640612843

Färber, A., Young, A. J., Ruban, A. V., Horton, P., and Jahns, P. (1997). Dynamics of xanthophyll-cycle activity in different antenna subcomplexes in the photosynthetic membranes of higher plants: the relationship between zeaxanthin conversion and nonphotochemical fluorescence quenching. Plant Physiol. 115, 1609-1618. doi: 10.1104/pp.115.4.1609

Goss, R., and Jakob, T. (2010). Regulation and function of xanthophyll cycle-dependent photoprotection in algae. Photosynth. Res. 106, 103-122. doi: $10.1007 /$ s11120-010-9536-x

Hager, A. (1969). Light dependent decrease of the pH-value in a chloroplast compartment causing the enzymatic interconversion of violaxanthin to zeaxanthin; relations to photophosphorylation. Planta 89, 224-243. doi: 10.1007/BF00385028

Havaux, M. (1998). Carotenoids as membrane stabilizers in chloroplasts. Trends Plant Sci. 3, 147-151. doi: 10.1016/S1360-1385(98)01200-X

Havaux, M., Bonfils, J. P., Lutz, C., and Niyogi, K. K. (2000). Photodamage of the photosynthetic apparatus and its dependence on the leaf developmental stage in the npq1 Arabidopsis mutant deficient in the xanthophyll cycle enzyme violaxanthin de-epoxidase. Plant Physiol. 124, 273-284. doi: $10.1104 /$ pp.124.1.273 
Havaux, M., Dall'Osto, L., and Bassi, R. (2007). Zeaxanthin has enhanced antioxidant capacity with respect to all other xanthophylls in Arabidopsis leaves and functions independent of binding to PSII antennae(1[C][W]). Plant Physiol. 145, 1506-1520. doi: 10.1104/pp.107.108480

Havaux, M., Dall'Osto, L., Cuine, S., Giuliano, G., and Bassi, R. (2004). The effect of zeaxanthin as the only xanthophyll on the structure and function of the photosynthetic apparatus in Arabidopsis thaliana. J. Biol. Chem. 279, 13878-13888. doi: 10.1074/jbc.M311154200

Havaux, M., Eymery, F., Porfirova, S., Rey, P., and Dormann, P. (2005). Vitamin E protects against photoinhibition and photooxidative stress in Arabidopsis thaliana. Plant Cell 17, 3451-3469. doi: 10.1105/tpc.105.037036

Havaux, M., and Niyogi, K. K. (1999). The violaxanthin cycle protects plants from photooxidative damage by more than one mechanism. Proc. Natl. Acad. Sci. U.S.A. 96, 8762-8767. doi: 10.1073/pnas.96.15.8762

Holt, N. E., Zigmantas, D., Valkunas, L., Li, X. P., Niyogi, K. K., and Fleming, G. R. (2005). Carotenoid cation formation and the regulation of photosynthetic light harvesting. Science 307, 433-436. doi: 10.1126/science.1105833

Holzwarth, A. R., Miloslavina, Y., Nilkens, M., and Jahns, P. (2009). Identification of two quenching sites active in the regulation of photosynthetic lightharvesting studied by time-resolved fluorescence. Chem. Phys. Lett. 483, 262-267. doi: 10.1016/j.cplett.2009.10.085

Horton, P., Ruban, A. V., and Wentworth, M. (2000). Allosteric regulation of the light-harvesting system of photosystem II. Philos. Trans. R. Soc. Lond. B Biol. Sci. 355, 1361-1370. doi: 10.1098/rstb.2000.0698

Jahns, P., Graf, M., Munekage, Y., and Shikanai, T. (2002). Single point mutation in the Rieske iron-sulfur subunit of cytochrome b(6)/f leads to an altered $\mathrm{pH}$ dependence of plastoquinol oxidation in Arabidopsis. FEBS Lett. 519, 99-102. doi: 10.1016/S0014-5793(02)02719-9

Jahns, P., Latowski, D., and Strzalka, K. (2009). Mechanism and regulation of the violaxanthin cycle: the role of antenna proteins and membrane lipids. Biochim. Biophys. Acta-Bioenerget. 1787, 3-14. doi: 10.1016/j.bbabio.2008.09.013

Jahns, P., and Miehe, B. (1996). Kinetic correlation of recovery from photoinhibition and zeaxanthin epoxidation. Planta 198, 202-210. doi: 10.1007/BF00206245

Jahns, P., Wehner, A., Paulsen, H., and Hobe, S. (2001). De-epoxidation of violaxanthin after reconstitution into different carotenoid binding sites of light-harvesting complex II. J. Biol. Chem. 276, 22154-22159. doi: 10.1074/jbc.M102147200

Kana, R., Kotabova, E., Kopecna, J., Trskova, E., Belgio, E., Sobotka, R., et al. (2016). Violaxanthin inhibits nonphotochemical quenching in light-harvesting antenna of Chromera velia. FEBS Lett. 590, 1076-1085. doi: $10.1002 / 1873-3468.12130$

Kirchhoff, H. (2014). Structural changes of the thylakoid membrane network induced by high light stress in plant chloroplasts. Philos. Trans. R. Soc. B Biol. Sci. 369:20130225. doi: 10.1098/rstb.2013.0225

Krause, G. H. (1988). Photoinhibition of photosynthesis. An evaluation of damaging and protective mechanisms. Physiol.Plant. 74, 566-574. doi: 10.1111/j.1399-3054.1988.tb02020.x

Krause, G. H., and Jahns, P. (2003). "Pulse amplitude modulated chlorophyll fluorometry and its application in plant science," in Light-Harvesting Antennas in Photosynthesis, eds B. R. Green and W. W. Parson (Dordrecht: Kluwer Academic Publishers), 373-399.

Krause, G. H., Vernotte, C., and Briantais, J. -M. (1982). Photoinduced quenching of chlorophyll fluorescence in intact chloroplasts and algae. Resolution into two components. Biochim. Biophys. Acta 679, 116-124. doi: 10.1016/0005-2728(82)90262-6

Kromdijk, J., Glowacka, K., Leonelli, L., Gabilly, S. T., Iwai, M., Niyogi, K. K., et al. (2016). Improving photosynthesis and crop productivity by accelerating recovery from photoprotection. Science 354, 857-861.doi: 10.1126/science.aai8878

Kyle, D. J., Ohad, I., and Arntzen, C. J. (1984). Membrane protein damage and repair: selective loss of a quinone-protein function in chloroplast membranes. Proc. Natl. Acad. Sci. U.S.A. 81, 4070-4074. doi: 10.1073/pnas.81.13.4070

Leitsch, J., Schnettger, B., Critchley, C., and Krause, G. H. (1994). Two mechanisms of recovery from photoinhibition in vivo: reactivation of photosystem II related and unrelated to D1 protein turnover. Planta 194, 15-21. doi: 10.1007/BF00201029
Leverenz, R. L., Sutter, M., Wilson, A., Gupta, S., Thurotte, A., de Carbon, C. B., et al. (2015). A 12 angstrom carotenoid translocation in a photoswitch associated with cyanobacterial photoprotection. Science 348, 1463-1466. doi: $10.1126 /$ science.aaa7234

Li, X.-P., Björkman, O., Shih, C., Grossman, A. R., Rosenquist, M., Jansson, S., et al. (2000). A pigment-binding protein essential for regulation of photosynthetic light harvesting. Nature 403, 391-395. doi: 10.1038/35000131

Li, X. P., Muller-Moule, P., Gilmore, A. M., and Niyogi, K. K. (2002). PsbSdependent enhancement of feedback de-excitation protects photosystem II from photoinhibition. Proc. Natl. Acad. Sci. U.S.A. 99, 15222-15227. doi: 10.1073/pnas.232447699

Li, Z., Peers, G., Dent, R. M., Bai, Y., Yang, S. Y., Apel, W., et al. (2016). Evolution of an atypical de-epoxidase for photoprotection in the green lineage. Nat. Plants 2:16140. doi: 10.1038/nplants.2016.140

Li, Z. R., Wakao, S., Fischer, B. B., and Niyogi, K. K. (2009). Sensing and responding to excess light. Annu. Rev. Plant Biol. 60, 239-260. doi: 10.1146/annurev.arplant.58.032806.103844

Macko, S., Wehner, A., and Jahns, P. (2002). Comparison of violaxanthin deepoxidation from the stroma and lumen sides of isolated thylakoid membranes from Arabidopsis: implications for the mechanism of de-epoxidation. Planta 216, 309-314. doi: 10.1007/s00425-002-0848-8

Miloslavina, Y., Wehner, A., Lambrev, P. H., Wientjes, E., Reus, M., Garab, G., et al. (2008). Far-red fluorescence: a direct spectroscopic marker for LHCII oligomer formation in non-photochemical quenching. FEBS Lett. 582, 3625-3631. doi: 10.1016/j.febslet.2008.09.044

Morosinotto, T., Caffarri, S., Dall'Osto, L., and Bassi, R. (2003). Mechanistic aspects of the xanthophyll dynamics in higher plant thylakoids. Physiol. Plant. 119, 347-354. doi: 10.1034/j.1399-3054.2003.00213.x

Muller, M. G., Lambrev, P., Reus, M., Wientjes, E., Croce, R., and Holzwarth, A. R. (2010). Singlet energy dissipation in the photosystem II light-harvesting complex does not involve energy transfer to carotenoids. Chemphyschem 11, 1289-1296. doi: 10.1002/cphc.200900852

Munekage, Y., Takeda, S., Endo, T., Jahns, P., Hashimoto, T., and Shikanai, T. (2001). Cytochrome b(6)f mutation specifically affects thermal dissipation of absorbed light energy in Arabidopsis. Plant J. 28, 351-359. doi: 10.1046/j.1365-313X.2001.01178.x

Naranjo, B., Mignee, C., Krieger-Liszkay, A., Hornero-Mendez, D., GallardoGuerrero, L., Javier Cejudo, F., et al. (2016). The chloroplast NADPH thioredoxin reductase C, NTRC, controls non-photochemical quenching of light energy and photosynthetic electron transport in Arabidopsis. Plant Cell Environ. 39, 804-822. doi: 10.1111/pce.12652

Nilkens, M., Kress, E., Lambrev, P. H., Miloslavina, Y., Müller, M., Holzwarth, A. R., et al. (2010). Identification of a slowly inducible zeaxanthin-dependent component of non-photochemical quenching of chlorophyll fluorescence generated under steady state conditions in Arabidopsis. Biochim. Biophys. Acta 1797, 466-475. doi: 10.1016/j.bbabio.2010.01.001

Niyogi, K. K., Grossman, A. R., and Bjorkman, O. (1998). Arabidopsis mutants define a central role for the xanthophyll cycle in the regulation of photosynthetic energy conversion. Plant Cell 10, 1121-1134. doi: $10.1105 /$ tpc.10.7.1121

Pan, X. W., Li, M., Wan, T., Wang, L. F., Jia, C. J., Hou, Z. Q., et al. (2011). Structural insights into energy regulation of light-harvesting complex CP29 from spinach. Nat. Struct. Mol. Biol. 18, 309-315. doi: 10.1038/nsmb.2008

Papadatos, S., Charalambous, A. C., and Daskalakis, V. (2017). A pahway for protective quenching in antenna proteins of Photosystem, II. Sci. Rep. 7:2523. doi: 10.1038/s41598-017-02892-w

Passarini, F., Wientjes, E., Hienerwadel, R., and Croce, R. (2009). Molecular basis of light harvesting and photoprotection in CP24 UNIQUE FEATURES OF THE MOST RECENT ANTENNA COMPLEX. J. Biol. Chem. 284, 29536-29546. doi: 10.1074/jbc.M109.036376

Pribil, M., Pesaresi, P., Hertle, A., Barbato, R., and Leister, D. (2010). Role of plastid protein phosphatase TAP38 in LHCII dephosphorylation and thylakoid electron flow. PLoS Biol. 8:e1000288. doi: 10.1371/journal.pbio.1000288

Reinhold, C., Niczyporuk, S., Beran, K., and Jahns, P. (2008). Short-term downregulation of zeaxanthin epoxidation in Arabidopsis thaliana in response to photo-oxidative stress conditions. Biochim. Biophys. Acta 1777, 462-469. doi: $10.1016 /$ j.bbabio.2008.03.002 
Ruban, A. V., Berera, R., Ilioaia, C., van Stokkum, I. H. M., Kennis, J. T. M., Pascal, A. A., et al. (2007). Identification of a mechanism of photoprotective energy dissipation in higher plants. Nature 450, 575-578. doi: 10.1038/nature06262

Ruban, A. V., and Horton, P. (1999). The xanthophyll cycle modulates the kinetics of nonphotochemical energy dissipation in isolated light-harvesting complexes, intact chloroplasts, and leaves of spinach. Plant Physiol. 119, 531-542. doi: 10.1104/pp.119.2.531

Ruban, A. V., Johnson, M. P., and Duffy, C. D. (2012). The photoprotective molecular switch in the photosystem II antenna. Biochim. Biophys. Acta 1817, 167-181. doi: 10.1016/j.bbabio.2011.04.007

Sacharz, J., Giovagnetti, V., Ungerer, P., Mastroianni, G., and Ruban, A. V. (2017). The xanthophyll cycle affects reversible interactions between PsbS and lightharvesting complex II to control non-photochemical quenching. Nat. Plants 3:16225. doi: $10.1038 /$ nplants.2016.225

Samuelsson, G., Lonneborg, A., Rosenqvist, E., Gustafsson, P., and Oquist, G. (1985). Photoinhibition and reactivation of photosynthesis in the cyanobacterium Anacystis nidulans. Plant Physiol. 79, 992-995. doi: 10.1104/pp.79.4.992

Sapozhnikov, D. I., Krasovskaya, T. A., and Maevskaya, A. N. (1957). Change in the interrelationship of the basic carotenoids of the plastids of green leaves under the action of light. Dokl. Akad. Nauk USSR 113, 465-467.

Schumann, T., Paul, S., Melzer, M., Doermann, P., and Jahns, P. (2017). Plant growth under natural light conditions provides highly flexible shortterm acclimation properties toward high light stress. Front. Plant Sci. 8:681. doi: $10.3389 /$ fpls.2017.00681

Shapiguzov, A., Ingelsson, B., Samol, I., Andres, C., Kessler, F., Rochaix, J.D., et al. (2010). The PPH1 phosphatase is specifically involved in LHCII dephosphorylation and state transitions in Arabidopsis. Proc. Natl. Acad. Sci. U.S.A. 107, 4782-4787. doi: 10.1073/pnas.0913810107

Tardy, F., and Havaux, M. (1997). Thylakoid membrane fluidity and thermostability during the operation of the xanthophyll cycle in higher-plant chloroplasts. Biochim. Biophys. Acta 1330, 179-193. doi: 10.1016/S0005-2736(97)00168-5

Thiele, A., Krause, G. H., and Winter, K. (1998). In situ study of photoinhibition of photosynthesis and xanthophyll cycle activity in plants growing in natural gaps of the tropical forest. Aust. J. Plant Physiol. 25, 189-195. doi: 10.1071/PP97119
Tikkanen, M., and Aro, E.-M. (2012). Thylakoid protein phosphorylation in dynamic regulation of photosystem II in higher plants. Biochim. Biophys. Acta 1817, 232-238. doi: 10.1016/j.bbabio.2011.05.005

Triantaphylides, C., and Havaux, M. (2009). Singlet oxygen in plants: production, detoxification and signaling. Trends Plant Sci. 14, 219-228. doi: 10.1016/j.tplants.2009.01.008

van Bezouwen, L. S., Caffarri, S., Kale, R. S., Kouril, R., Thunnissen, A. W. H., Oostergetel, G. T., et al. (2017). Subunit and chlorophyll organization of the plant photosystem II supercomplex. 3:17080. doi: 10.1038/nplants.2017.80

Wehner, A., Storf, S., Jahns, P., and Schmid, V. H.R. (2004). De-epoxidation of violaxanthin in light-harvesting complex I proteins. J. Biol. Chem. 279, 26823-26829. doi: 10.1074/jbc.M402399200

Wei, X. P., Su, X. D., Cao, P., Liu, X. Y., Chang, W. R., Li, M., et al. (2016). Structure of spinach photosystem II-LHCII supercomplex at 3.2 angstrom resolution. Nature 534, 69-74. doi: 10.1038/nature18020

Wilson, A., Ajlani, G., Verbavatz, J. M., Vass, I., Kerfeld, C. A., and Kirilovsky, D. (2006). A soluble carotenoid protein involved in phycobilisomerelated energy dissipation in cyanobacteria. Plant Cell 18, 992-1007. doi: 10.1105/tpc.105.040121

Xu, P., Tian, L., Kloz, M., and Croce, R. (2015). Molecular insights into Zeaxanthin-dependent quenching in higher plants. Sci. Rep. 5:13679. doi: 10.1038/srep13679

Yamamoto, H. Y., Nakayama, T. O.M., and Chichester, C. O. (1962). Studies on the light and dark interconversions of leaf xanthophylls. Arch. Biochem. Biophys. 97, 168-173. doi: 10.1016/0003-9861(62)90060-7

Conflict of Interest Statement: The authors declare that the research was conducted in the absence of any commercial or financial relationships that could be construed as a potential conflict of interest.

Copyright (c) 2017 Kress and Jahns. This is an open-access article distributed under the terms of the Creative Commons Attribution License (CC BY). The use, distribution or reproduction in other forums is permitted, provided the original author(s) or licensor are credited and that the original publication in this journal is cited, in accordance with accepted academic practice. No use, distribution or reproduction is permitted which does not comply with these terms. 